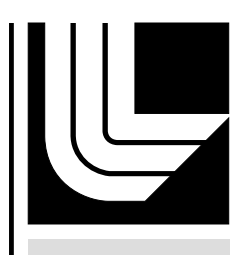

LAWRENCE LIVERMORE N A TION A L LABORATORY

\title{
Analytical SuperSTEM for extraterrestrial materials research
}

J. P. Bradley, Z. R. Dai

September 18, 2009

Meteoritics and Planetary Science 
This document was prepared as an account of work sponsored by an agency of the United States government. Neither the United States government nor Lawrence Livermore National Security, LLC, nor any of their employees makes any warranty, expressed or implied, or assumes any legal liability or responsibility for the accuracy, completeness, or usefulness of any information, apparatus, product, or process disclosed, or represents that its use would not infringe privately owned rights. Reference herein to any specific commercial product, process, or service by trade name, trademark, manufacturer, or otherwise does not necessarily constitute or imply its endorsement, recommendation, or favoring by the United States government or Lawrence Livermore National Security, LLC. The views and opinions of authors expressed herein do not necessarily state or reflect those of the United States government or Lawrence Livermore National Security, LLC, and shall not be used for advertising or product endorsement purposes. 


\title{
Analytical SuperSTEM for extraterrestrial materials research
}

\author{
John. P. Bradley and Zu Rong Dai
}

Institute of Geophysics and Planetary Physics

Lawrence Livermore National Laboratory

Livermore CA, 94551

To be submitted to:

Meteoritics and Planetary Science 


\begin{abstract}
Electron-beam studies of extraterrestrial materials with significantly improved spatial resolution, energy resolution and sensitivity are enabled using a $300 \mathrm{keV}$ SuperSTEM scanning transmission electron microscope with a monochromator and two spherical aberration correctors. The improved technical capabilities enable analyses previously not possible. Mineral structures can be directly imaged and analyzed with single-atomiccolumn resolution, liquids and implanted gases can be detected, and UV-VIS optical properties can be measured. Detection limits for minor/trace elements in thin $(<100 \mathrm{~nm}$ thick) specimens are improved such that quantitative measurements of some extend to the sub-500 ppm level. Electron energy-loss spectroscopy (EELS) can be carried out with $0.10-0.20 \mathrm{eV}$ energy resolution and atomic-scale spatial resolution such that variations in oxidation state from one atomic column to another can be detected. Petrographic mapping is extended down to the atomic scale using energy-dispersive $x$-ray spectroscopy (EDS) and energy-filtered transmission electron microscopy (EFTEM) imaging. Technical capabilities and examples of the applications of SuperSTEM to extraterrestrial materials are presented, including the UV spectral properties and organic carbon K-edge fine structure of carbonaceous matter in interplanetary dust particles (IDPs), x-ray elemental maps showing the nanometer-scale distribution of carbon within GEMS (glass with embedded metal and sulfides), the first detection and quantification of trace Ti in GEMS using EDS, and detection of molecular $\mathrm{H}_{2} \mathrm{O}$ in vesicles and implanted $\mathrm{H}_{2}$ and $\mathrm{He}$ in irradiated mineral and glass grains.
\end{abstract}




\section{Introduction}

Electron microscopy is a key analytical discipline in meteoritics and planetary science, as well as in materials science, because of the unique capability to simultaneously measure structure (morphology, grain size, crystallography) and composition. In the late 1960s and 1970s, during and after Apollo, the meteoritics community made widespread use of the electron microprobe and scanning electron microscope (SEM) (e.g. Adler et al., 1970; Keil et al., 1970; Wood et al., 1970). The transmission electron microscope (TEM) began to be widely utilized during the 1970s (e.g. Bibring et al., 1970; Buseck, 1977; Buseck and Veblen, 1978). Each of these electron-beam instruments is optimized for specific types of analysis, although there is significant overlap in capabilities. The electron microprobe is configured with crystal spectrometers for wavelength-dispersive x-ray spectroscopy (WDS) and mapping of thick-flat specimens, the SEM for secondary and backscattered electron imaging of bulk and thick-flat specimens in conjunction with EDS, and the TEM for imaging and diffraction studies of thin (electron transparent) specimens.

The analytical scanning transmission electron microscope (STEM), a hybrid instrument that combines imaging and spectroscopic capabilities of SEM and TEM, first appeared in meteoritics in the early 1980s (e.g. Fraundorf, 1981). It has since become the most widely used type of TEM in meteoritics (Bradley et al., 1983; Bradley et al., 1989; Germani et al., 1990; Keller and MacKay, 1997; Rietmeijer, 2002; Joswiak et al., 2009; Leroux et al., 2009). Until recently, there had been no fundamental advances in the design of TEM/STEM instruments since the first commercial instruments were produced. The earliest TEMs were optimized for structural analysis (imaging and diffraction), and later instruments were also configured for microanalysis with focused nanoprobes and $\mathrm{x}$ ray and electron (energy-loss) spectrometers. (At least one TEM was manufactured with crystal spectrometers). Most instruments used tungsten (W) or lanthanum hexaboride $\left(\mathrm{LaB}_{6}\right)$ electron emitters with useful probe sizes of $\sim 10 \mathrm{~nm}$, solid-state $\mathrm{x}$-ray (EDS) detectors with relatively small collection angles ( $\sim 0.1$ steradians), illumination systems with serious aberrations and significant levels of stray radiation, poor vacuum systems susceptible to water vapor and hydrocarbon contamination, and unstable specimen 
platform/goniometer stages. Better vacuum systems enabled higher brightness field emission (FE) sources that are in widespread use today, but microanalysis with high spatial resolution is still compromised by specimen drift, contamination, and relatively low (x-ray) count rates. Although dedicated STEMs (like the VG series of instruments) offered significantly better performance, the full analytical potential of TEM/STEMs was limited by their basic design.

A new generation of electron microscope known as SuperSTEM has recently become available for materials and meteoritics (Fig. 1). The term SuperSTEM refers to a STEM instrument with a monochromator and at least one spherical aberration $\left(\mathrm{C}_{\mathrm{s}}\right)$ corrector (Brown and Bleloch, 2000). The SuperSTEM at Lawrence Livermore National Laboratory (LLNL), funded in part by NASA's Sample Return and Laboratory Instrument Development Program (SRLIDAP), is an FEI high-base Titan equipped with dual $\left(\mathrm{C}_{\mathrm{s}}\right)$ correctors, one for sub-Ångstrom TEM imaging and the other for sub-Ångstrom STEM imaging and spectroscopy using a sub-Angstrom $(0.8 \AA$ diameter $)$ probe. Other innovations include isolation of the microscope column in a climate controlled enclosure (Fig. 1b), a much improved dry vacuum system, a high stability specimen stage $(<2 \AA$ drift/minute specification), a sub-Angstrom diameter electron probe, improved EDS capabilities and a high (energy) resolution electron energy-loss spectrometer/energy filter.

In this paper, we describe the current capabilities of a modern SuperSTEM installed at LLNL. We also describe examples of how these new capabilities have been and are being applied to the analysis of extraterrestrial materials, in particular, complex finegrained materials, amorphous materials, implanted materials and inclusions. Finally, we discuss how thee capabilities may be integrated with other analysis techniques to provide a more complete and detailed understanding of the nature of the finest-grained extraterrestrial materials.

\section{Advances enabled by SuperSTEM}

In this section we describe the following attributes of the LLNL SuperSTEM: the monochromator, spherical aberration $\left(\mathrm{C}_{\mathrm{s}}\right)$ correctors, EDS and electron energy loss/ EFTEM capabilities. 
The electron gun monochromator: The monochromator is a device that reduces energy spread in the primary electron beam. Two types of monochromator, the Wienfilter and Omega filter, are now used in STEM (Brink et al., 2003; Mukai et al., 2007). Both disperse electrons emitted from the tip perpendicular to the optic axis. An energy slit is used to limit the energy distribution of the electrons, such that the energy spread in the beam is controlled by the strength of the dispersion and the width of the slit. The most important impact of the monochromator is the improvement in energy resolution for electron energy-loss spectroscopy (EELS) and electron energy-loss near edge structure (ELNES) analysis (Fig. 2). EELS and ELNES are particularly sensitive to detection of light elements ( $\mathrm{Li}, \mathrm{Be}, \mathrm{C}, \mathrm{N}, \mathrm{O}$ ). ELNES is analogous to (synchrotron) $\mathrm{x}$-ray absorption near edge structure (XANES), in that it can be used to determine the valence state(s) and electronic structure of specific atoms in a specimen. However, ELNES offers 2-3 orders of magnitude better spatial resolution than XANES allowing higher precision analyses on fine-grained phases, although energy spread in the electron beam, lens aberrations and high-voltage instabilities have historically limited ELNES energy resolution to the 0.5$2.0 \mathrm{eV}$ range, depending on the type of electron source. XANES, in contrast, is capable of $0.1 \mathrm{eV}$ energy resolution. Energy resolution using these spectroscopies is critically important because the intrinsic fine structure of absorption/ionization edges is on the order of $\sim 0.1 \mathrm{eV}$ (Egerton, 1996). The advent of the monochromator has provided relatively routine ELNES energy resolution of $\sim 0.1 \mathrm{eV}$ like that of XANES (Mook and Kruit, 2000; Botton et al., 2003; Dai et al., 2005; Erni et al., 2005). We have achieved EELS energy resolution of $70 \mathrm{meV}(0.07 \mathrm{eV})$ at $200 \mathrm{keV}$ and $160 \mathrm{meV}$ at $300 \mathrm{keV}$ using the monochromator and (Gatan) high-resolution energy filter (HRGIF) (Fig. 2).

Figure 3 compares high-resolution (HR) EELS and XANES data acquired at similar energy resolution $(0.1-0.15 \mathrm{eV})$. The monochromator enables examination of energyloss near edge fine structure with $\sim 0.1 \mathrm{eV}$ resolution, although a significant drawback of the monochromator is a decrease in beam current and edge signal strength resulting from the energy slit in the beam path. At energy losses below $\sim 500 \mathrm{eV}$ the EELS and ELNES are not significantly degraded, but at losses above $500 \mathrm{eV}$ longer acquisition times are required to offset the lower monochromated beam currents. Longer acquisition times mean lower energy resolution (Fig. 2c). 
The spherical aberration $\left(C_{s}\right)$ corrector: Lens aberrations are one of the biggest obstacles in improving image resolution and forming high-brightness, sub-Ångstrom diameter electron probes for spectroscopic analysis. During the past decade, improved understanding of electron optics theory, more robust computational capabilities, computer control of electron optical columns and better power supplies have led to the first commercially available spherical Cs correctors for imaging and analysis. There are several $\mathrm{C}_{\mathrm{s}}$ corrector designs (for both objective and condenser lenses) most of which use combinations of magnetic lenses (quadruples, octapoles, sextapoles, and additional lenses) fitted between the second condenser and objective lens of the microscope (Freitag et al., 2005; Sawada et al., 2005). The practical effects of $\mathrm{C}_{\mathrm{s}}$ correction include better spatial resolution, smaller probes, higher probe intensities, greater contrast, stronger spectroscopic signal and greater sensitivity. Examples of the improved spatial resolution, direct structural imaging at the sub-Ångstrom scale, and spectroscopy, single-atomiccolumn (HR) EELS and EDS measurements, are shown in Figures. 4 \& 5). The principle disadvantage of a $\mathrm{C}_{\mathrm{s}}$-corrected probe is that the higher probe intensities can cause rapid irradiation damage in specimens, especially organic materials.

Energy-dispersive X-ray spectroscopy: EDS detectors are an integral component of most electron-beam instruments (electron microprobe, SEM, TEM and STEM). They are the most rapid and convenient method for measuring and mapping the compositions of specimens (Figs. $5 b \& 6 \& 7$ ). EDS of thin (S)TEM specimens offers superior spatial resolution over bulk or thick-flat specimens (typically examined using SEM or electron microprobe) because electron beam spreading is minimized in thin specimens such that (x-ray) spatial resolution approaches the diameter of the incident electron probe. Practical STEM EDS detection limits for most elements are on the order of $\sim 1000 \mathrm{ppm}$ under optimal experimental conditions, although high precision quantitative measurements at $<2000 \mathrm{ppm}$ are rare. The x-ray detector on the LLNL SuperSTEM (and other modern STEM instruments) offers significantly improved performance as a result of the better, cleaner vacuum system that enable longer spectral acquisition times with less specimen contamination. An X-twin pole-piece also enables the detector to be positioned closer to the specimen ( $9 \mathrm{~mm}$ as opposed to $14 \mathrm{~mm}$ ) providing a higher solid angle ( 0.3 steradians rather than 0.16 steradians). Other SuperSTEM attributes that 
positively impact EDS are the larger active detector surface area $\left(30 \mathrm{~mm}^{2}\right.$ as opposed to $10 \mathrm{~mm}^{2}$ ), the high stability, actuator-controlled specimen stage and the $\mathrm{C}_{\mathrm{s}}$-corrected subÅngstrom nanoprobe. The LLNL SuperSTEM is capable of atomic-scale EDS measurements for major (and even minor) elements under the appropriate experimental conditions (e.g. Fig. 5c). Quantitative EDX measurements can be performed down to the 250-500 ppm level (Fig. 7, Table 1). This regime of quantification has traditionally been the domain of WDS using the electron microprobe and $x$-ray fluorescence (XRF). EDS will never compete with synchrotron XRF at the level of $\sim 200 \mathrm{ppm}$ and below, in part, because electrons excite Bremsstrahlung that leads to high backgrounds even in thin films (e.g. Fig. 7).

Energy-filtered (EF) imaging. The energy filter (electron energy-loss spectrometer) is a device that filters electrons transmitted through a thin specimen as a function of their energy (Brink et al., 2003). Using a slit of variable width, the energy-filtered electrons can be selected for imaging with a specific energy loss and energy bandwidths. Contrast in EFTEM images can reflect compositional variation (e.g. Fig. 8), similar to the contrast observed in x-ray maps (e.g. Fig. 6). EFTEM offers several advantages over x-ray mapping. First, in addition to its sensitivity to the light elements, image acquisition time is usually less than one minute, allowing chemical imaging at high magnification and with spatial resolution better than $1 \mathrm{~nm}$. Because of the short acquisition times, the requirement of high specimen stability that is a prerequisite for $\mathrm{x}$-ray maps, acquired over minutes or hours, is less important with EFTEM imaging. Second, just as EELS can probe the local electronic structure of specimens, energy-selected images showing variations in local electronic structure can be formed (Batson et al., 1994; Kimoto et al., 1999; Wang et al., 1999). Third, quantitative chemical images with spatial resolution of $\sim 1 \mathrm{~nm}$ and sensitivity for some elements at the $\sim 1 \%$ level are possible (Elbert et al., 2001). However, contrast in energy-filtered images can be more difficult to interpret than x-ray maps because of the dynamical nature of the process itself; contrast can be altered by energy shifts, energy resolution and high exponential background underlying ionization edges, as well as specimen thickness and plural scattering (Hofer et al., 2003; Schaffer et al., 2006). 


\section{Applications of SuperSTEM to meteoritics}

Optical properties: The monochromator on a modern SuperSTEM reduces the width of the zero-loss peak in an electron energy-loss spectrum (Fig. 2) so that features with energy losses as low as $\sim 0.2 \mathrm{eV}$ can be detected. The so-called "low-loss region" between 0 and $20 \mathrm{eV}$ contains information about band gaps, intra band and inter band transitions, including optical transitions in ultraviolet (UV) and visible spectral regions. The ability to perform optical spectroscopy using low-loss EELS is important because, (a) it is one of the few spectroscopic measurement methods common to observational astronomy and laboratory studies of meteoritic materials (Bradley et al., 1992; 2005), and (b) optical data can be obtained from the finest grained meteoritic materials down to the atomic scale.

In one example of the use of low-loss EELS to link observational astronomy and laboratory studies, the UV spectral characteristics of IDPs were investigated using lowloss EELS (Bradley et al., 2005). We detected a 5.7 eV (2175 $\AA$ ) feature associated with carbonaceous organic matter and GEMS) in IDPs (Fig. 9). The position of the feature corresponds to the astronomical $2175 \AA$ extinction feature, or "blue bump", the strongest spectral signature of interstellar dust observed by astronomers. Several of the grains we analyzed have non-solar isotopic compositions suggesting that they were derived from the presolar molecular cloud or interstellar medium (ISM). The grains responsible for the $2175 \AA$ feature in the ISM are believed to be carbonaceous, so the finding of a carbonaceous carrier in IDPs was not unexpected. However, detection of a similar feature in glass-rich GEMS in IDPs was unexpected. We suggested that these GEMS may contain organic carbonaceous components. Acid dissolution experiments showed that GEMS contain significant quantities of carbonaceous matter, but the experiments could not distinguish whether the carbonaceous matter was within or on the surfaces of GEMS as coatings (Brownlee et al., 2000; Dai et al., 2002). Compositional mapping using the SuperSTEM reveals that carbonaceous material is indeed carried within the interiors of GEMS (Fig. 6).

Detection of gases and water: Gasses and liquids trapped in tiny quantities in meteoritic materials are challenging to analyze by any method. Although gases and liquids are volatile and susceptible to mass loss under electron irradiation, it is possible to 
spectroscopically detect them using the SuperSTEM. The features manifest themselves in the low-loss region where low beam currents and spectral acquisition times $(<2$ seconds) are required to avoid signal oversaturation. Monochromated low-loss EELS was used to detect oxygen and water $\left(\mathrm{H}_{2} \mathrm{O}\right)$ within IDPs (Fig. 10). Oxygen and water were detected in fluid inclusions within vesiculated alumosilicate glass in an IDP W7013E-17 (Erni et al., 2005). The glass contains nanometer-sized voids that were likely formed by heating during atmospheric entry (Fig. 10a). Despite their probable secondary origin, the vesicles enable us to demonstrate that low-loss EELS is capable of detecting molecular oxygen and water in fluid inclusions in geological materials. Lowloss spectra reveal a peak at $8.6 \mathrm{eV}$ and absorption bands between 12 and $16 \mathrm{eV}$ reflecting vibrational excitation states, i.e. excitons of $\mathrm{O}_{2}$ molecules (Fig. 10b). The presence of oxygen gas is supported by the corresponding oxygen K-edge fine structure (Fig. 10c). Peaks at 539 and $542 \mathrm{eV}$ are caused by $1 \mathrm{~s} \rightarrow 2 \mathrm{p} \sigma^{*}$ transitions and additional small peaks at 534 and $536 \mathrm{eV}$ can be assigned to $\mathrm{H}_{2} \mathrm{O} \sigma^{*}$ peaks (Fig. 10d).

Characterization of silicate glasses: Silicate glasses are commonly present in meteorites, (e.g. within chondrules), micrometeorites, IDPs and in the comet 81P/Wild 2 ("Wild 2") sample returned to Earth by the Stardust mission. The provenance of glasses can be extremely difficult to assess because, unlike crystalline silicates, there are fewer constraints on their compositions and mechanisms of formation. The SuperSTEM at LLNL is being used to intensively examine glasses in GEMS (glass with embedded metal and sulfides). A brief introduction to GEMS, their astronomical relevance and the questions surrounding them are presented here prior to descriptions of SuperSTEM analyses that provide better characterization of these astrophysically important objects.

GEMS are submicrometer amorphous silicate grains that are abundant constituents of the most cosmically primitive class of meteoritic materials, chondritic porous (CP) IDPs. There is widespread interest among meteoriticists and astronomers in GEMS because most of the condensed atoms in the solar system were carried within amorphous silicate grains immediately prior to the collapse of the solar nebula. Amorphous silicates are among the most abundant grains in the ISM (Sandford, 1996; Draine, 2003). They are believed to originate primarily in the circumstellar outflows of oxygen-rich stars. During their lifetimes in diffuse and dense regions of the ISM, they are subjected to a variety of 
processes (e.g. shocks, irradiation, accumulation of organic mantles, coagulation) leading to amorphization and, frequently, complete destruction. Amorphous silicate dust grains with optical properties similar to those of interstellar silicates have recently been observed concentrated within the outer accretion disks of other young stars (Van Boekel et al, 2004). This dust is likely interstellar dust that has escaped the significant heating, recrystallization, vaporization and condensation that occurs within hotter, inner disk environments. GEMS are suspected to be examples of this outer accretion disk material from our own solar nebula. It has been confirmed, via laboratory isotope measurements, that some GEMS in CP IDPs were interstellar amorphous silicates incorporated into dust in our solar system (Messenger et al., 2003; Floss et al., 2006; Min et al., 2008). Indeed, recent modeling comparisons to astronomical silicate dust spectra show remarkable agreement between contemporary ISM dust and GEMS that were incorporated into CP IDPs long ago (Min et al., 2007; 2008; Bradley \& Ishii, 2008).

The details of how the glasses in GEMS formed and were processed remains the subject of debate largely due to the challenges of interpreting composition and morphology of amorphous materials. Several different scenarios have been proposed for the formation of GEMS, and SuperSTEM analyses including low-loss EELS, high sensitivity EDX and petrographic mapping are being applied to determine which of these hypotheses is most consistent with GEMS' characteristics. Bradley (1994) proposed a single population of GEMS that have been exposed to grain processing, predominantly irradiation, since their formation in circumstellar environments and their $\sim 10^{8}$ year lifetimes in the ISM. Others have proposed that there are multiple populations of GEMS, some formed in presolar circumstellar environments, but most formed in the solar nebula (Keller \& Messenger, 2004; 2007; 2008; Keller et al., 2005). They propose solar system formation of GEMS by late stage, non-equilibrium condensation or shock melting followed by transport to the comet forming regions by, for example, bipolar outflows (Keller \& Messenger, 2004). These formation scenarios are unlikely because heating experiments indicate that GEMS were formed at relatively low temperatures (Brownlee et al., 2005). Further complicating matters, laboratory experiments have shown that "GEMS-like" material can be produced by multiple processes including irradiation (Bradley 1994; Keller \& MacKay, 1997), impact and shock (Ishii et al., 2008), and vapor 
phase condensation (Rietmeijer et al. 2002, Davoisne et al. 2006). Any or all of these processes, as well as other processes like grain sticking and coagulation, are implicated in the formation of dust as it passes through interstellar and molecular cloud environments. GEMS would have been subjected to the same processing.

The return of the Stardust mission in 2006 stimulated further interest in GEMS because glassy silicates resembling GEMS are abundant in the impact tracks in Stardust aerogel. Since GEMS are the most abundant silicate in CP IDPs, their presence or absence in the comet Wild 2 sample is a key arbitrator of whether there is a relationship between CP IDPs and Wild 2 grains. Initially, it appeared that GEMS were abundant in the Stardust sample (Keller et al., 2006; Zolensky et al., 2006), but subsequent TEM measurements, in conjunction with light gas-gun shots using Stardust grain analogues, suggest that much if not all of this "GEMS-like" material may have been produced by melting, ablation and mixing of Wild 2 grains with aerogel during hypervelocity capture (Ishii et al., 2008). Other glasses in the impact tracks may be indigenous to comet Wild 2 and related to chondrule mesostasis in chondritic meteorites (Tomeoka et al., 2008). Irradiation effects: Space is a harsh environment and extraterrestrial materials are subject to a number of space weathering processes including irradiation. Evidence of irradiation in small particulate extraterrestrial samples is often taken as an indication of residence time in space. Hydrogen and helium are highly abundant in the solar system and comprise the dominant implanting species in many energy regimes.

If GEMS were indeed exposed to prolonged periods of irradiation in space, one of the theories for their processing history, then they should contain implanted $\mathrm{H}$ and $\mathrm{He}$, assuming they are not subsequently lost due to frictional heating during atmospheric entry. Noble gas measurements have shown that IDPs contain saturation doses of implanted He (Pepin et al., 2001). Using monochromated low-loss EELS we have taken the first steps to determine whether implanted $\mathrm{H}$ and $\mathrm{He}$ can be detected in individual GEMS by irradiating polished thick-flat specimens of San Carlos olivine with $5 \mathrm{keV} \mathrm{H}_{2}{ }^{+}$ and $\mathrm{He}^{+}$ions at fluences of $\sim 1 \mathrm{X} 10^{19} \mathrm{H}^{+} / \mathrm{cm}^{2}$ and $7.5 \times 10^{18} \mathrm{He}^{+} / \mathrm{cm}^{2}$. In both cases, irradiation exposure produced a bubble-rich amorphous rim 75 to $100 \mathrm{~nm}$ thick (Figs. 11a \& 11b). Low-loss spectra from the $\mathrm{H}^{+}$-irradiated rim show a $\mathrm{H}-\mathrm{K}$ core loss edge at 12 $\mathrm{eV}$ as well as additional features attributable to $\mathrm{H}_{2} \mathrm{O}$ and $\mathrm{OH}^{-}$(Fig. 11c), whereas the 
spectra from the He-irradiated rim only show a He-K edge at $\sim 22 \mathrm{eV}$ (Fig. 11d). Lowloss spectra from GEMS grains in relatively unheated IDPs are being collected by SuperSTEM at LLNL.

In addition to implantation, irradiation imparts changes in the relative cation abundances in silicate minerals. For example, $\mathrm{Mg}$ and $\mathrm{Ca}$ depletions have been observed in solar wind irradiated amorphous surfaces on lunar soil silicate grains and IDPs (Bradley, 1994; Topanni et al., 2006: Keller and MacKay, 1997). Ion implantation experiments duplicating the irradiation regimes typical of the solar wind and supernova shocks in the ISM confirm both amorphisation and/or chemical changes. For example, when silicates like olivine, pyroxene and some feldspars are irradiated $\mathrm{Mg}, \mathrm{Al}, \mathrm{Ca}$ and Ti segregate within the irradiated surface: Irradiation of crystalline pyroxenes produces an amorphous glass that is depleted in $\mathrm{Mg}$ and $\mathrm{Ca}$ and enriched in $\mathrm{Al}$ and Ti relative to the un-irradiated pyroxene, while irradiation of ( $\mathrm{Al}$ and $\mathrm{Ca}$-free) olivine produces glass that is depleted in Mg (Bradley et al., 1996; Topanni et al., 2006; Davoisne, 2008). Under most geochemical conditions (e.g. shock melting and condensation) $\mathrm{Mg}, \mathrm{Al}, \mathrm{Ca}$ and $\mathrm{Ti}$ are expected to follow one another. Atomic mass differences and solid state environment (e.g. coordination and bond strengths) are likely responsible for the differential response of refractory cations under $(0.1-10 \mathrm{keV})$ irradiation conditions typical of the solar wind and supernova shocks. However, the magnitude of chemical effects depends on the irradiation regime. For example, under $(\mathrm{MeV})$ irradiation conditions typical of galactic cosmic rays we found that olivine (forsterite) rapidly amorphizes without any significant change in composition (Bringa et al., 2007).

New quantitative EDS measurements of $\mathrm{Mg}, \mathrm{Al}, \mathrm{Ca}$ and $\mathrm{Ti}$ abundances in GEMS are consistent with a history of radiation exposure. The bulk compositions of GEMS are within a factor of 2-3 chondritic (solar) but $\mathrm{Mg}$ and $\mathrm{Ca}$ are systematically depleted (Keller and Messenger, 2004; Bradley and Ishii, 2008). Table 1 lists the compositions of seven GEMS. These SuperSTEM data differ from previous EDS measurements from GEMS in that there are several orders of magnitude more counts in the spectra; at least $10^{5}$ counts in the Al peaks, $10^{6}$ counts in the Si peaks, $10^{4}$ counts in the Ca peaks and $10^{3}$ counts in the Ti peaks. S, Cr, Mn, Fe and Ni are within $\pm 40 \%$ of solar abundances, in accordance with previous GEMS measurements. $\mathrm{Fe}, \mathrm{S}$ (and Ni), that are carried in FeNi 
metal and sulfide inclusions, are all systemically depleted from solar abundances by similar magnitudes (60-80\%). $\mathrm{Mg}, \mathrm{Al}, \mathrm{Ca}, \mathrm{Ti}, \mathrm{Cr}$ and $\mathrm{Mn}$ are carried in the glass matrices of GEMS. Relative to solar abundances, $\mathrm{Cr}$ and $\mathrm{Mn}$ are within $\pm 30 \%, \mathrm{Mg}$ and $\mathrm{Ca}$ are depleted (60\% and 40\% respectively) and $\mathrm{Al}$ and $\mathrm{Ti}$ are enriched (190\% and $220 \%$ respectively). (Ti is the most heavily depleted refractory element in the gas phase in the diffuse ISM (O'Donnell and Mathis, 1997), indicating that it is concentrated in grains.

The $\mathrm{Al}$ and (new) Ti data showing enrichments, together with the $\mathrm{Mg}$ and $\mathrm{Ca}$ data confirming depletions, provide additional evidence in support of prior irradiation exposure histories for GEMS with at least three important caveats. First, the starting (pre-exposure) $\mathrm{Mg}, \mathrm{Al}, \mathrm{Ca}$ and Ti compositions of GEMS are assumed to have been solar but are, in fact, unknown. Second, Table 1 contains high-precision Al and Ti measurements from only 7 GEMS, but a much larger population of GEMS needs to be measured before firm conclusions can be drawn about the significance of minor and trace element abundances. Third, the response of sulfide minerals to irradiation has not yet been experimentally investigated. Table 1 (and Figure 7) establish that quantitative measurements of minor and trace elements like Ti are feasible, and future SuperSTEM measurements on a larger populations of GEMS are likely to generate an improved understanding of the processes involved in their formation and evolution.

Petrographic mapping: Understanding the petrography of mineral assemblages is a cornerstone of geochemistry in general and of meteoritics in particular. Nanoscale mapping is assuming an important role in the largely unexplored area of IDP and meteorite petrography (Figs. $6 \& 8$ ), as well as in the petrography of other fine-grained meteoritic materials (Dai and Bradley, 2005; Floss et al. 2004; Floss et al. 2006). Mapping with micrometer-scale resolution is a common capability of electron microprobes and SEMs. Mapping with nanometer-scale resolution is possible using STEM, but it requires a very clean high-vacuum environment, a stable specimen stage (ideally $<10 \AA \mathrm{drift} /$ minute) and a high-brightness nanoprobe. Mapping with spatial resolution of single atomic columns $(<5 \AA)$ has been demonstrated using the SuperSTEM.

The nature of carbon and organic matter in IDPs and other meteoritic materials is highly relevant to astrobiology. Synchrotron XANES and now HREELS are providing useful 
information about the molecular speciation of meteoritic organics (Fig. 3) Figure 6 shows an example in which compositional mapping at the nanometer scale yielded hitherto unknown information about petrographic distribution of carbon that reveals an astronomically intriguing feature. This "single" GEMS grain is revealed to be composed of at least three grains, each having acquired a carbonaceous mantle prior to coagulation into the object shown in Fig. 6a. In other words, carbon mapping has revealed evidence of an aggregation/coagulation episode that predates the origin of the GEMS grain. Phenomena like accumulation of organic mantles, grain-grain collisions and coagulation are believed to be central to the processing of grains in the interstellar medium and molecular cloud environments (Draine, 2003; Stepnik et al., 2003; Jones, 2005), suggesting that GEMS with such mantles formed prior to the collapse of the solar nebula. Petrographic maps are obtained using energy-filtered imaging, $\mathrm{x}$-ray mapping or both (Figs. 6 \& 8). Energy-filtered imaging offers higher collection efficiency, rapid image acquisition (1-60 seconds), and high sensitivity for light elements (C, N and O); however, quantitative information is challenging, but entirely possible, to extract. Extended data acquisition times (30-60 minutes) are required for X-ray mapping because of low x-ray collection efficiency. In addition, higher electron irradiation doses are required and sensitivities for light elements (B, C, N, O) are lower. Sample degradation can be a major issue using a Cs-corrected probe and specimen drift compensation software is mandatory. The highest spatial resolution x-ray maps are typically acquired with lower counting statistics, while those with counting statistics sufficient for quantitative $\mathrm{x}$-ray mapping are typically acquired at lower spatial resolution. The choice of a particular petrographic mapping method depends on the stability and grains size of the material to be analyzed.

Summary and future developments

SuperSTEM enables electron-beam studies of meteoritic materials with improved resolution and sensitivity. Mineral stucture can be directly imaged and spectroscopically analyzed with atomic resolution, optical properties can be measured at the atomic scale and liquids and implanted gases can be detected. Electron spectroscopy can be performed with energy resolution comparable to synchrotron XANES $(\sim 100 \mathrm{meV})$ but 
with atomic scale sensitivity and spatial resolution. EDS detection limits are improved and petrographic mapping is extended down to the atomic scale using EDS and EFTEM. The increase in analytical capabilities is accompanied by an increased total beam dose and potentially more electron irradiation damage to some geological materials. However, unprecedented computer control of gun and lens operating parameters mean that $\mathrm{C}_{\mathrm{s}^{-}}$ corrected instruments like the SuperSTEM can be configured for low-dose microscopy without significantly compromising overall analytical performance (Evans et al., 2008). Software for low-dose microscopy is being contemplated for the LLNL SuperSTEM with the goal of using low-dose microscopy routinely to examine beam-sensitive meteoritic materials like organic compounds and silicate glasses. Both are important constituents of chondritic materials (Bradley, 2004; Matrajt et al., 2008)

Continuing improvements in EELS and ELNES are enabling new kinds of measurements, for example, quantitative determination of the $\mathrm{Ti}^{3+} / \mathrm{Ti}^{4+}$ ratio the comet Wild 2 CAI "Inti" (Chi et al., 2009). These improvements may be particularly useful in the low-loss $(0-20 \mathrm{eV})$ region where the monochromator enables detection of the lightest elements $(\mathrm{H}$ and $\mathrm{He})$ as well as features related to the optical and electronic properties of specimens (e.g. Figs. 9-11). Because ionization cross sections are $\sim 10^{2}-10^{4}$ higher in the low-loss region, considerably lower beam currents are needed, which is a major advantage with beam sensitive materials. However, the low-loss region is dominated by plasmon scattering that is highly sensitive to changes in physical and chemical (electronic) properties of the specimen. As such, features of interest are typically superimposed on a dynamically changing background (Egerton, 1996). Detailed modeling of the low-loss spectrum in conjunction with the use of standard samples are required to enable us to fully exploit low-loss spectroscopy. For example, with development of background management algorithms, it should be possible to map the petrographic distribution of implanted $\mathrm{H}$ and $\mathrm{He}$ using low-loss spectroscopy and EFTEM.

The capabilities of SuperSTEM will have the most impact in combination with other analytical techniques. We are increasingly emphasizing an integrated analytical approach to the characterization of meteoritic materials, whereby a single specimen is sequentially analyzed using a combination of methods, e.g. SEM, SuperSTEM, nanoSIMS and 
synchrotron spectroscopy (Bradley et al., 2005; Floss et al., 2006). One of the key enabling technologies for integrated microanalysis is the Focused Ion Beam instrument (FIB) that enables "site-specific" sampling of bulk and particulate specimens (Graham et al., 2008). The FIB also enables nanoscale etching, patterned deposition of metal masks and machining to stabilize or isolate specific regions of a specimen for subsequent analyses (Ishii et al., 2009; Matzel et al., 2009). Integrated microanalysis that includes SuperSTEM will maximize the mineralogical, elemental, isotopic, and spectroscopic information extracted from a single specimen. For particulate extraterrestrial samples such as IDPs and Stardust grains where the total amount of available mass is very small, this is especially advantageous. 


\section{Acknowledgement:}

This research was supported by NASA SRLIDAP and Cosmochemistry program grants

to J.P.B. Portions of this work were performed under the auspices of the U.S.

Department of Energy by Lawrence Livermore National Laboratory under Contract DEAC52-07NA27344. We thank M. Chi and M. Barfels for input and D. Joswiak for a detailed review. 


\section{References:}

Adler, I.; Walter, L. S.; Lowman, P. D.; Glass, B. P.; French, B. M.; Philpotts, J. A.; Heinrich, K. J. F.; Goldstein, J. I. (1970) Electron microprobe analysis of Apollo 11 lunar samples. Geochim. Cosmochim. Acta (Supp.) 1, 87-92.

Anders, E. and Ebihara, M. (1982) Solar system abundances of the elements. Geochim. Cosmochim. Acta 46, 2363-2380.

Batson, P. E., Browning, N. D. and Muller, D. A. (1994) Electron energy-loss near-edge structure studies at the atomic level: reliability of the spatial difference technique. $M S A$ Bulletin 24, 371-378.

Bibring, J. P.; Duraud, J. P.; Durrieu, L.; Jouret, C.; Maurette, M. and Meunier, R. (1970) Ultrathin Amorphous Coatings on Lunar Dust Grains. Science 175, 753-755.

Botton, G. A., Lazar, S., Wu, M. -Y., Tichelaar and Zandbergen, H. W. (2003) Materials science applications of a monochromated TEM. Microsc. Microanal. (Supp.) 9, 112113.

Bradley, J. P. (1994) Chemically anomalous, preaccretionally irradiated grains in interplanetary dust from comets. Science 265, 925-929.

Bradley, J. P. and Ishii, H. A. (2008) Comment on the shape and composition of interstellar silicate grains. $A \& A, \mathbf{4 8 6}, 781-784$.

Bradley, J. P., Brownlee, D. E. and Veble, D. R. (1983) Pyroxene whiskers and platelets in interplanetary dust - Evidence of vapour phase growth. Nature 301, 473-477. 
Bradley, J. P. Dai, Z. R., Erni, R., Browning, N. D., Graham, G., Weber, P., Smith, Julie, J. Hutcheon, I., Ishii, H., Bajt, S., Floss, C., Stadermann, F.and Sandford, S. (2005) An Astronomical $2175 \AA$ Feature in Interplanetary Dust Particles. Science 307, 244-247.

Bradley, J. P., Dukes, C., Baragiola, R., McFadden, L., Johnson, R. E. and Brownlee, D. E. (1996), Radiation Processing and the Origins of Interplanetary Dust. Lunar Planet. Sci. XXVII, 149-150.

Bradley, J.P., Germani, M.S., and Brownlee, D.E., (1989) Automated Thin-Film Analyses of Anhydrous Interplanetary Dust Particles in the Analytical Electron Microscope, Earth Planet. Sci. Lett. 93, 1-13.

Bradley, J. P., Germani, M.S., and Humecki, H. J. (1992) Combined infrared and analytical electron microscope studies of interplanetary dust particles. Ap. J. 394, 643651.

Bringa, E. M., Kucheyev, S. O., Loeffler, M. J., Baragiola, R. A., Tielens, A. G. G. M., Dai, Z. R., Graham, G., Bajt, S., Bradley, J. P., Dukes, C. A., Felter, T. E., Torres, D. F. and Van Breugel, W. (2007) Energetic Processing of Interstellar Silicate Grains by Cosmic Rays. Ap. J. 662, $372-378$.

Brink, H. A., Barfels, M. M. G., Burgner, R. P. and Edwards, B. N. (2003) A sub-50 eV spectrometer and energy filter for use in combination with $200 \mathrm{keV}$ monochromated (S)TEMs. Ultramicroscopy 96, 367-384.

Brown, L. M. and Bleloch, A. L. (2000) In Microscopy and Microanalysis 2000. G. W. Bailey, S. McKernan, R. L. Price, S. D. Walck, P.-M, Charest, R. Gauvin Eds.) (Springer-Verlag, New York, 2000), pp. 98-99.

Brownlee, D. E., Joswiak, D. J., Bradley, J. P., Gezo, J. C. and Hill, H. G. M. (2000) 
Spatially resolved acid dissolution of IDPs: The state of carbon and the abundance of diamonds in the dust. Lunar Planet. Sci. XXXI, Abs. \# 1921.

Brownlee, D. E., Joswiak, D. J., Bradley, J. P., Matrajt, G. and Wooden, D. E. (2005) Cooked GEMS - Insights into the hot origins of crystalline silicates in circumstellar disks and the cold origins of GEMS. Lunar Planet. Sci. XXXVI, Abs. \#2391.

Brownlee, Don; Tsou, Peter; Aléon, Jérôme; Alexander, Conel M. O.'D.; Araki, Tohru; Bajt, Sasa; Baratta, Giuseppe A.; Bastien, Ron; Bland, Phil; Bleuet, Pierre; Borg, Janet; Bradley, John P.; Brearley, Adrian; Brenker, F.; Brennan, Sean; Bridges, John C.; Browning, Nigel D.; Brucato, John R.; Bullock, E.; Burchell, Mark J.; Busemann, Henner; Butterworth, Anna; Chaussidon, Marc; Cheuvront, Allan; Chi, Miaofang; Cintala, Mark J.; Clark, B. C.; Clemett, Simon J.; Cody, George; Colangeli, Luigi; Cooper, George; Cordier, Patrick; Daghlian, C.; Dai, Zurong; D'Hendecourt, Louis; Djouadi, Zahia; Dominguez, Gerardo; Duxbury, Tom; Dworkin, Jason P.; Ebel, Denton S.; Economou, Thanasis E.; Fakra, Sirine; Fairey, Sam A. J.; Fallon, Stewart; Ferrini, Gianluca; Ferroir, T.; Fleckenstein, Holger; Floss, Christine; Flynn, George; Franchi, Ian A.; Fries, Marc; Gainsforth, Z.; Gallien, J.-P.; Genge, Matt; Gilles, Mary K.; Gillet, Philipe; Gilmour, Jamie; Glavin, Daniel P.; Gounelle, Matthieu; Grady, Monica M.; Graham, Giles A.; Grant, P. G.; Green, Simon F.; Grossemy, Faustine; Grossman, Lawrence; Grossman, Jeffrey N.; Guan, Yunbin; Hagiya, Kenji; Harvey, Ralph; Heck, Philipp; Herzog, Gregory F.; Hoppe, Peter; Hörz, Friedrich; Huth, Joachim; Hutcheon, Ian D.; Ignatyev, Konstantin; Ishii, Hope; Ito, Motoo; Jacob, Damien; Jacobsen, Chris; Jacobsen, Stein; Jones, Steven; Joswiak, David; Jurewicz, Amy; Kearsley, Anton T.; Keller, Lindsay P.; Khodja, H.; Kilcoyne, A. L. David; Kissel, Jochen; Krot, Alexander; Langenhorst, Falko; Lanzirotti, Antonio; Le, Loan; Leshin, Laurie A.; Leitner, J.; Lemelle, L.; Leroux, Hugues; Liu, Ming-Chang; Luening, K.; Lyon, Ian; MacPherson, Glen; Marcus, Matthew A.; Marhas, Kuljeet; Marty, Bernard; Matrajt, Graciela; McKeegan, Kevin; Meibom, Anders; Mennella, Vito; Messenger, Keiko; Messenger, Scott; Mikouchi, Takashi; Mostefaoui, Smail; Nakamura, Tomoki; Nakano, T.; Newville, M.; Nittler, Larry R.; Ohnishi, Ichiro; Ohsumi, Kazumasa; Okudaira, Kyoko; 
Papanastassiou, Dimitri A.; Palma, Russ; Palumbo, Maria E.; Pepin, Robert O.; Perkins, David; Perronnet, Murielle; Pianetta, P.; Rao, William; Rietmeijer, Frans J. M.; Robert, François; Rost, D.; Rotundi, Alessandra; Ryan, Robert; Sandford, Scott A.; Schwandt, Craig S.; See, Thomas H.; Schlutter, Dennis; Sheffield-Parker, J.; Simionovici, Alexandre; Simon, Steven; Sitnitsky, I.; Snead, Christopher J.; Spencer, Maegan K.; Stadermann, Frank J.; Steele, Andrew; Stephan, Thomas; Stroud, Rhonda; Susini, Jean; Sutton, S. R.; Suzuki, Y.; Taheri, Mitra; Taylor, Susan; Teslich, Nick; Tomeoka, Kazu; Tomioka, Naotaka; Toppani, Alice; Trigo-Rodríguez, Josep M.; Troadec, David; Tsuchiyama, Akira; Tuzzolino, Anthony J.; Tyliszczak, Tolek; Uesugi, K.; Velbel, Michael; Vellenga, Joe; Vicenzi, E.; Vincze, L.; Warren, Jack; Weber, Iris; Weisberg, Mike; Westphal, Andrew J.; Wirick, Sue; Wooden, Diane; Wopenka, Brigitte; Wozniakiewicz, Penelope; Wright, Ian; Yabuta, Hikaru; Yano, Hajime; Young, Edward D.; Zare, Richard N.; Zega, Thomas; Ziegler, Karen; Zimmerman, Laurent; Zinner, Ernst; Zolensky, Michael (2006) Comet 81P/Wild 2 under a microscope. Science 314, 17111713.

Burban, J. P., Chi, M., Masiel, D.J., Bradley, J. P., Jiang, B., Stahlberg, H. and Browning, N. D. (2009) Structural variability of edge dislocations in $\mathrm{SrTiO}_{3}$ low-angle [001] tilt grain boundary. J. Mat Res. (in press).

Buseck, P. R. (1977) Pallasite meteorites - Mineralogy, petrology and geochemistry. Geochim. Cosmochim. Acta 41, 711-721.

Buseck, P. R. and Veblen, D. R. (1978) Trace elements, crystal defects and high resolution electron microscopy. Geochim. Cosmochim. Acta, 42, 669-678.

Chi, M., Ishii, H. A., Dai' Z. R., Simon, S., Joswiak, D. J., Browning, D. J. and Bradley, J. P. (2009) The origin of refractory minerals in Comet 81P/Wild 2. Geochim. Cosmochim. Acta. Submitted. 
Dai, R. R. and Bradley, J. P. (2005) Origin and properties of GEMS (Glass with Embedded Metal and Sulfides). In Chondrites and the Protoplanetary Disk (Eds. A. N. Krot, E. R. D. Scott \& B. Reipurth), ASP Conf. Series, Vol 134, 668-674.

Dai, Z. R., Bradley, J. P., Erni, R. and Browning, N. D. (2005) High-resolution electron energy-loss spectroscopy (HREELS) using a monochromated TEM/STEM. Lunar Planet. Sci. XXXVI, Abs. \# 2110.

Dai, Z. R., Bradley, J. P., Joswiak, D. J., Brownlee, D. E., Hill, H. G. M. and Genge, M. J. (2002) Possible in situ formation of meteoritic nanodiamonds in the early Solar System. Nature 418, 157-159.

Davoisne, C., Djouadi, Z., Leroux, H., D'Hendecourt, L., Jones, A. P.; Deboffle, D (2006) The origin of GEMS as deduced from the microstructural evolution of amorphous silicates with annealing. $A \& A$ 448, L1-L4.

Davoisne, C., Leroux, H., Frère, M. Gimblot, J., Gengembre, L., Djouadi, Z., Ferreiro, V., D'Hendecourt, L. and Jones, A. (2008) Chemical and morphological evolution of a silicate surface under low-energy ion irradiation $A \& A$ 482, 541-548.

Draine, B. (2003) Interstellar dust grains. Ann. Rev. of Astron. Astrophys 41, 241-289.

Egerton, R. F. (1996) Electron Energy-loss Spectroscopy in the Electron Microscope. (Plenum Press, New York). pp. 485.

Elbert, D. C., Ilton, E. S. and Veblen, D. R. (2001) EFTEM Valence Imaging of Minerals I: Ferric and Ferrous Iron. Eleventh Annual V. M. Goldschmidt Conference, May 20-24, 2001, Hot Springs, Virginia, abstract no. 3893. 
Erni, R., Browning, N, D., Dai, Z. and Bradley. J. P. (2005) Analysis of extraterrestrial particles using monochromated electron energy-loss spectroscopy (EELS). Micron 36, 369-379.

Evans, J. E., Hetherington, C., Kirkland, A., Chang, L. Y., Stahlberg, H. and browning, D. D. (2008) Low-dose aberration corrected cryo-electron microscopy of organic specimens. Ultramicroscopy 108, 1636-1644.

Floss, C., Stadermann, F. J., Bradley, J. P., Dai, Z. R., Bajt, S. and Graham, G. (2004) Carbon and Nitrogen Isotopic Anomalies in an Anhydrous Interplanetary Dust Particle. Science 303, 1355-1358.

Floss, C., Stadermann, F. J., Bradley, J. P., Dai, Z. R., Bajt, S. Graham, G. and Lea, A. S. (2006) Geochim Cosmochim Acta 70, 2371-2382.

Fraundorf, P. (1981) Interplanetary dust in the transmission electron microscope Diverse materials from the early solar system. Geochim Cosmochim Acta 45, 915-937.

Freitag, B., Kujawa, S., Mul, P.M., Ringnalda, J. and Tiemeijer, P. C. (2005) Breaking the spherical and chromatic aberration barrier in transmission electron microscopy. Ultramicroscopy 102, 209-214.

Germani, M.S., and Bradley, J.P., "Automated Point Count Analysis of Interplanetary Dust Particle Thin-Sections," Lunar Planet Sci. XIX, 385-386 (1990).

Graham, G. A., Teslich, N. E., Kearsley, A. T., Stadermann, F. J., Stroud, R. M., Dai, Z., Ishii, H. A., Hutcheon, I. D., Bajt, S., Snead, C. J., Weber, P. K. and Bradley, J. P. (2008) Applied focused ion beam techniques for sample preparation of astromaterials for integrated nanoanalysis. Met. Planet. Sci. 43, 561-569. 
Hitchcock, A. P. and Mancini, D. C. (1993) http://unicorn.mcmaster.ca/corex/name$\underline{\text { list.html (unpublished). }}$

Hofer, F., Grogger, W., Kothleitner, G. and Warbichler (2003) Analaytical electron microscopy in materials and biological sciences. Microsc. Microanal. 9 (Suppl.) 12-13.

Ishii, H. A., Bradley, J. P., Dai, Z. R., Chi, M., Kearsley, A. T., Burchell, M. J., Browning, N. D. and Molster, F. (2008) Comparison of Comet 81P/Wild 2 Dust with Interplanetary Dust from Comets. Science 319, 447-449.

Ishii, H. A.; Joswiak, D.; Bradley, J. P.; Teslich, N.; Matzel, J.; Hutcheon, I. D.; Brownlee, D.; Matrajt, G.; MacPherson, G.; McKeegan, K. D. (2009) Enabling Al-Mg Isotopic Measurements on Comet Wild 2's Micro-CAIs. Lunar Planet. Sci. XXXX, Abs. \#2288.

Jones, Anthony P. (2005) Dust formation, propagation and survival in the ISM. Proceedings of the dusty and molecular universe: a prelude to Herschel and ALMA, 2729 October 2004, Paris, France. Ed. by A. Wilson. ESA SP-577, Noordwijk, Netherlands: ESA Publications Division, ISBN 92-9092-855-7, 2005, p. 239 - 244.

Joswiak, D. J., Brownlee, D. ., Matrajt, G., Westphal, A. J. and Snead, C. (2009) Kosmochloric Ca-rich pyroxenes and $\mathrm{FeO}-$ rich olivines (Kool grains) and associated phases in Stardust tracks and chondritic porous interplanetary dust particles: possible precursors to Type II FeO-rich chondrules. Met. Planet Sci. (this volume).

Keil, K., Prinz, M. and Bunch, T. E. (1970) Mineral Chemistry of Lunar Samples, Science 167, 597-599.

Keller L. P. \& MacKay D. S. (1997) The nature and origin of rims on lunar soil grains. Geochim. Cosmochim. Acta A 61, 2331-2241. 
Keller L. P. and Messenger (2004) On the origin of GEMS. Lunar Planet. Sci. XXXV Abs. \#1985.

Keller L. P. and Messenger (2008) Coordinated Chemical and Isotopic Studies of GEMS Grains in IDPs. Lunar Planet. Sci. XXXVIIII Abs. \#1391.

Keller L. P. and Messenger (2007) Coordinated Chemical and Isotopic Studies of IDPs: Comparison of Circumstellar and Solar GEMS Grains. MAPS 42, 5297.

Keller, L. P., Bajt, S., Baratta, G. A., Borg, J., Bradley, J. P., Brownlee, D. E., Busemann, H., Brucato, J. R., Burchell, M. Colangeli, L., D'Hendecourt, L., Djouadi, Z., Ferrini, G., Flynn, G., Franchi, I. A., Fries, M., Grady, M. M., Graham, G. A., Grossemy, F., Kearsley, A., Matrajt, G., Nakamura-Messenger, K., Mennella, V., Nittler, L., Palumbo, M. E., Stadermann, F. J., Tsou, P., Rotundi, A., Sandford, S. A., Snead, C., Steele, A., Wooden, D. and Zolensky, M. (2006) Infrared Spectroscopy of Comet 81P/Wild 2 Samples Returned by Stardust. Science 314, 1728-1730.

Keller, L. P.; Messenger, S.; Christoffersen, R. (2005) GEMS Revealed: Spectrum Imaging of Aggregate Grains in Interplanetary Dust. Lunar Planet. Sci. XXXVI Abs. \# 2088.

Kimoto, K., Kobayashia, K., Aoyama, T. and Mitsui, Y. (1999) Analyses of composition and chemical shift of silicon oxynitride film using energy-filtering transmission electron microscope based spatially resolved electron energy loss spectroscopy. Micron 30, 121127.

Leroux, H, Roskosz, M. and Jacob, D. (2009) Oxidation state of iron and extensive redistribution of sulfur in thermally modified Stardust particles. Geochim. Cosmochim. Acta 73, 767-777. 
Matzel, J., Ishii, H., Joswiak, D. J., Hutcheon, I., Bradley, J. P., Brownlee, D. E., Weber, P., Ramon, E., Simon, J., Teslich, N., Matrajt, G., McKeegan, K. D. and MacPherson' G. (2009) $\mathrm{Mg}$ isotope measurements of a Stardust CAI: no evidence of ${ }^{26} \mathrm{Al}$. MAPS (submitted).

Matrajt, G., Ito, M., Wirick, S., Messenger, S., Brownlee, D. E., Joswiak, D., Flynn, G., Sandford, S.; Snead, C.; Westphal, A. (2008) Carbon investigation of two Stardust particles: A TEM, NanoSIMS, and XANES study. Met. Planet. Sci. 43, 315-334.

Messenger S., Keller L. P., Stadermann F. J., Walker R. M., Zinner E., Samples of Stars Beyond the Solar System: Silicate Grains in Interplanetary Dust (2003) Science 300, 105-108.

Min M., Waters L. B. F. M., de Koter A., Hovenier J. W., Keller L. P. and MarkwickKemper F. (2007) The shape and composition of interstellar silicate grains. $A \& A \mathbf{4 6 2}$, 667-676.

Min M., Waters L. B. F. M., de Koter A., Hovenier J. W., Keller L. P. and MarkwickKemper F. (2008) Erratum -The shape and composition of interstellar silicate grains. $A \& A, \mathbf{4 8 6}, 779-780$.

Mook, H. W. and Kruit, P. (2000). Construction and characterization of the fringe field monochromator for a field emission gun. Ultramicroscopy 81, 129-139.

Mook, H. W. Batson, P. E. and Kruit, P. (1999) In Electron Microscopy and Analysis, 1999 Ed. C. J. Kiely (Institute of Physics, Bristol) Vol 161, pp 223-226.

Mukai, T., Inami, W., Omoto, K., Kaneyama, T., Tomita, T., Tsuno, K., Terauchi, M., Tsuda, K., Satoh, Y., Naruse, M., Honda, T., Tanaka M. (2007) Performance of a monochromator for a $200 \mathrm{kV}$ analytical electron microscope. Microsc. Microanal. 13, 1242-1243. 
O’Donnell, J. E. and Mathis, J. S. (1997) Dust grain size distribution and the abundance of refractory elements in the diffuse interstellar medium. Ap. J. 479, 806-817.

Pepin, R. O., Palma, R. L. and Schlutter, D. J. (2001) Noble gases in interplanetary dust particles. II. Excess helium-3 in cluster particles and modeling constraints on interplanetary dust particle exposures to cosmic-ray irradiation. Met. \& Planet. Sci. 36, 1515-1534.

Rietmeijer F. J. M., Hallenbeck S. L., Nuth J. A. and Karner J. M. (2002) Amorphous Magnesiosilicate Smokes Annealed in Vacuum: The Evolution of Magnesium Silicates in Circumstellar and Cometary Dust. Icarus 156, 269-286.

Sandford, S. A. (1996) The inventory of interstellar materials available for the formation of the solar system. MAPS 31, 440-476.

Sawada H, Tomita T, Naruse M, Honda T, Hambridge P, Hartel P, Haider M, Hetherington C, Doole R, Kirkland A, Hutchison J, Titchmarsh J and Cockayne D. (2005) Experimental evaluation of a spherical aberration-corrected TEM and STEM. $J$ Electron Microsc. 54, 119-121.

Schaffer, B., Kothleitner, G. and Grogger, W. (2006) EFTEM spectrum imaging at highenergy resolution. Ultramicroscopy 106, 1129-1138.

Stepnik, B., Abergel, A., Bernard, J.-P., Boulanger, F., Cambrésy, L., Giard, M., Jones, A. P., Lagache, G., Lamarre, J.-M., Meny, C., Pajot, F., Le Peintre, F., Ristorcelli, I., Serra, G.; and Torre, J.-P. (2003) Evolution of dust properties in an interstellar filament. $A \& A$ 398, p.551-563

Tomeoka, K. Tomioka, N. and Ohnishi, I. (2008) Silicate minerals and Si-O glass in comet Wild 2 samples: Transmission electron microscopy. MAPS 43, 273-284. 
Topanni, A. Dukes, C., Baragiola, R. and Bradley, J. P. (2006) Segregation of Mg, Ca, $\mathrm{Al}$ and Ti in silicates during ion irradiation. Lunar Planet. Sci. XXXVII, Abs. 2056.

van Boekel, R., Min, M., Lienert, Ch., Waters, L. B. F. M. et al. (2004) The building blocks of planets within the 'terrestrial' region of protoplanetary disks. Nature 432, 479482.

Wang, Z. L. , Bentley, J. and Evans, N. D. (1999) Mapping the Valence States of Transition Metal Elements Using Energy-Filtered Transmission Electron Microscopy. $J$. Phys. Chem. B. 103, 751-753.

Wirick, S., Flynn, G. J., Keller, L., Nakarmura-Messenger, K., Sandford, S., Zolensky, M., Peltzer, C and Jacobsen, C. (2009) Evidence for changes in 81P/Wild 2 organic matter since collection and comparison of 81P/Wild 2 and IDP organic matter to access the thermal effects of aerogel capture. Lunar Planet. Sci. XXXX, Abs. \# 1366.

Wood, J. A., Dickey, J. S. Jr., Marvin, U. B. and Powell, B. N. (1970) Lunar Anorthosites. Science 167, 602-604.

Zolensky, Michael E.; Zega, Thomas J.; Yano, Hajime; Wirick, Sue; Westphal, Andrew J.; Weisberg, Mike K.; Weber, Iris; Warren, Jack L.; Velbel, Michael A.; Tsuchiyama, Akira; Tsou, Peter; Toppani, Alice; Tomioka, Naotaka; Tomeoka, Kazushige; Teslich, Nick; Taheri, Mitra; Susini, Jean; Stroud, Rhonda; Stephan, Thomas; Stadermann, Frank J.; Snead, Christopher J.; Simon, Steven B.; Simionovici, Alexandre; See, Thomas H.; Robert, François; Rietmeijer, Frans J. M.; Rao, William; Perronnet, Murielle C.; Papanastassiou, Dimitri A.; Okudaira, Kyoko; Ohsumi, Kazumasa; Ohnishi, Ichiro; Nakamura-Messenger, Keiko; Nakamura, Tomoki; Mostefaoui, Smail; Mikouchi, Takashi; Meibom, Anders; Matrajt, Graciela; Marcus, Matthew A.; Leroux, Hugues; Lemelle, Laurence; Le, Loan; Lanzirotti, Antonio; Langenhorst, Falko; Krot, Alexander N.; Keller, Lindsay P.; Kearsley, Anton T.; Joswiak, David; Jacob, Damien; Ishii, Hope; Harvey, Ralph; Hagiya, Kenji; Grossman, Lawrence; Grossman, Jeffrey N.; Graham, 
Giles A.; Gounelle, Matthieu; Gillet, Philippe; Genge, Matthew J.; Flynn, George; Ferroir, Tristan; Fallon, Stewart; Ebel, Denton S.; Dai, Zu Rong; Cordier, Patrick; Clark, Benton; Chi, Miaofang; Butterworth, Anna L.; Brownlee, Donald E.; Bridges, John C.; Brennan, Sean; Brearley, Adrian; Bradley, John P.; Bleuet, Pierre; Bland, Phil A.; Bastien, Ron (2006) Mineralogy and Petrology of Comet 81P/Wild 2 Nucleus Samples, Science 314, $1735-1738$. 


\section{Figure Captions}

Figure 1: The $300 \mathrm{keV}$ Titan SuperSTEM at Lawrence Livermore National Laboratory funded by NASA's SRLIDAP program. (a) Electron optical column with monochromator and dual spherical aberration $\left(\mathrm{C}_{\mathrm{s}}\right)$ correctors ( $\sim 3.5 \mathrm{~m}$ in height). (b) During operation the column is enclosed in a vibration-free, climate controlled "box". Temperature variation within the room is limited to less than $\pm 1^{\circ} \mathrm{C}$ /hour. (c) The microscope is isolated from the user and operated remotely from an adjoining room.

Figure 2: Electron energy-loss spectroscopy using a monochromator. (a) Zero-loss peak at $300 \mathrm{keV}$ and acquisition time of 1 second. (b) Zero-loss peak at $200 \mathrm{keV}$ with 1 second acquisition time. (c) Dependence of energy resolution on spectrum acquisition time at $200 \mathrm{keV}$.

Figure 3: Comparison of (HR)EELS and XANES C-K edges obtained at $\sim 0.1 \mathrm{eV}$ energy resolution. (a) $200 \mathrm{keV}$ high resolution (HR) EELS carbon-K edges obtained from IDP "Pupi" (U220GCA) using the monochromator and high-resolution energy filter. (b) XANES carbon-K edges obtained from amorphous carbon (top red trace), IDP L2009*E6 (middle black traces) and ordered graphite (lower red plot). XANES data from Wirick et al., 2009. (c) - (e) Exemplar brightfield TEM images showing locations from which HREELS spectra 1, 3 and 5 in (a) were obtained.

Figure 4: (a) $\mathrm{C}_{\mathrm{s}}$-corrected $300 \mathrm{keV}$ image of silicon in [211] orientation showing " $\mathrm{Si}$ dumbbells" spaced $1.4 \AA$ apart. (b) Fast Fourier Transform of image of (a) demonstrating sub-Ångström resolution. (c) [-12-10] atomic resolution image of a pyrrhotite grain in a Stardust track in aerogel. Dashed line shows unit cell and arrows show bright Fe-rich atomic columns. S-rich atomic columns are less intense and grey while the higher-Z Fe-rich columns are white. (d) Schematic diagram of atom columns projected along the [-12-10] crystallographic direction for pyrrhotite. 
Figure 5: Demonstration of atomic-resolution imaging, electron spectroscopy and (EDS) chemical profiling of a defect in a synthetic perovskite $\left(\mathrm{SrTiO}_{3}\right)$ standard. (a) Z-contrast dark field STEM image of a dislocation core in $\mathrm{SrTiO}_{3}$. (b) High-resolution (HR) EELS spectra from single atomic columns. ELNES examination shows only $\mathrm{Ti}^{4+}$ (4 peaks attributable to $\mathrm{Ti}^{4+}$ ligand field splitting) in bulk $\mathrm{SrTiO}_{3}$ (blue trace), and predominantly $\mathrm{Ti}^{3+}$ (2 peaks) within the dislocation core (red trace). The decrease in fine structure on the O-K edge (red trace) within the dislocation core is attributable to depletion of $\mathrm{Sr}^{2+}$. (c) Single atomic column resolution x-ray line profile across alternating $\mathrm{Ti}$ and $\mathrm{Sr}$ columns in $\mathrm{SrTiO}_{3}$ (Burban et al., 2009).

Figure 6: Brightfield (a and e) and darkfield (c) images of GEMS in CP IDPs with corresponding $\mathrm{C}-\mathrm{K}$ edge $\mathrm{x}$-ray maps ( $\mathrm{b}, \mathrm{d}$ and $\mathrm{f}$ ) showing the distribution of carbon associated with the GEMS.

Figure 7: Minor/trace element analysis in the SuperSTEM using advanced solid-state detector technology. Energy dispersive x-ray spectrum from a GEMS grain in CP IDP U2070A-2B. The spectrum was collected at an energy resolution of $130 \mathrm{eV}$ (defined at the Mn Ka edge) with 80\% dead time over 100 minutes. (a) Full 0-10 keV GEMS spectrum. $\mathrm{Cu}$ peaks are systems peaks (from the TEM grid and unrelated to specimen composition. (b) Vertically expanded spectrum showing $\mathrm{Ca}, \mathrm{Ti}, \mathrm{Mn}, \mathrm{Cr}$ and $\mathrm{Ni}$ peaks. (c) Spectrum from NBS 610 thin-film standard glass (61 elements at 500 ppm) collected at maximum energy resolution (130 eV at $\mathrm{Mn} \mathrm{K}-\alpha)$ with $80 \%$ dead time over 100 minutes. Considerably shorter acquisition times are feasible with lower dead times and lower energy resolution.

Figure 8: (a) Brightfield TEM image of an oxide grain boundary region in a Ni alloy electrode material. (b) $\mathrm{Ni}-\mathrm{M}_{2,3}$ core scattering edge. (c) Residual (net) $\mathrm{Ni}-\mathrm{M}_{2,3}$ edge following background subtraction. (d) Energy-filtered image formed from the residual $\mathrm{Ni}-\mathrm{M}_{2,3}$. Spatial resolution is $\sim 1 \mathrm{~nm}$, image acquisition time was 3 seconds. 
Figure 9: Comparison of astronomical UV extinction features with laboratory UV and low-loss EELS features. (a) The $2175 \AA$ interstellar extinction feature from two stars $\zeta$ and $\varepsilon$ Persei. (b) The profile as derived from 13 stars. Continuous line is the best fit Lorentzian. (c) Photo-absorption spectrum from partially-recrystallized hydroxylated amorphous magnesium silicate $\left(\mathrm{Mg}_{2} \mathrm{SiO}_{4}[\mathrm{OH}]_{\mathrm{n}}\right)$. (d) Low-loss EELS spectrum from (electron) irradiation damaged talc $\left(\mathrm{Mg}_{3} \mathrm{Si}_{4} \mathrm{O}_{10}[\mathrm{OH}]_{2}\right)$. (e) Low-loss EELS spectrum from (organic) carbon in IDP L2047 D23. (f) Low-loss EELS spectrum from GEMS in W7013 E17. Vertical scale in (a) is magnitude, and in (b) through (f) normalized logarithmic. Data from Bradley et al., 2005.

Figure 10: (a) High-angle annular darkfield (HAADF) image of a vesiculated aluminosilicate glass grain in IDP W7013E-17. (b) Low-loss spectra from silicate glass and one of the voids in the glass. The feature at $\sim 8.6 \mathrm{eV}$ is consistent with molecular $\mathrm{O}_{2}$ in the void, and the fine structure between 12 and $20 \mathrm{eV}$ is due to oxygen excitons. Weak features at 534 and $536 \mathrm{eV}$ are due to $\mathrm{H}_{2} \mathrm{O}$, and the feature at $\sim 21.5 \mathrm{eV}$ may be due to helium. (c) Oxygen core-loss K-edge spectra from glass matrix and a vesicle. (d) The EELS difference spectrum from the spectra (c) compared with a low-energy inner shell EELS O $\mathrm{O}_{2}$ spectrum (from Hitchcock and Mancini (1993). Data from Erni et al., 2005.

Figure 11. (a) and (b) $200 \mathrm{keV}$ brightfield images of the surface of San Carlos olivine irradiated with $\mathrm{H}$ and $\mathrm{He}$ ions. (c) and (d) Low-loss EELS spectra collected sequentially from within the bulk olivine substrate (blue traces) into the irradiated rims (green and red traces). In (c), features due to $\mathrm{OH}^{-}, \mathrm{H}_{2} \mathrm{O}$ and $\mathrm{H}_{2}$ are evident in the spectra from the $\mathrm{H}_{2}{ }^{+}$ irradiated rim. There is also a pronounced shift $(\sim 2 \mathrm{eV})$ in the position of the volume plasmon maxima. In (d), the He-K edge feature at $\sim 22 \mathrm{eV}$ is evident in the spectra from the $\mathrm{He}^{+}$irradiated rim. There is a minimal shift in the position of the volume plasmon. 


\section{Table 1.}

Quantitative energy-dispersive x-ray analyses (element/Si) of 7 GEMS in 2 CP IDPs

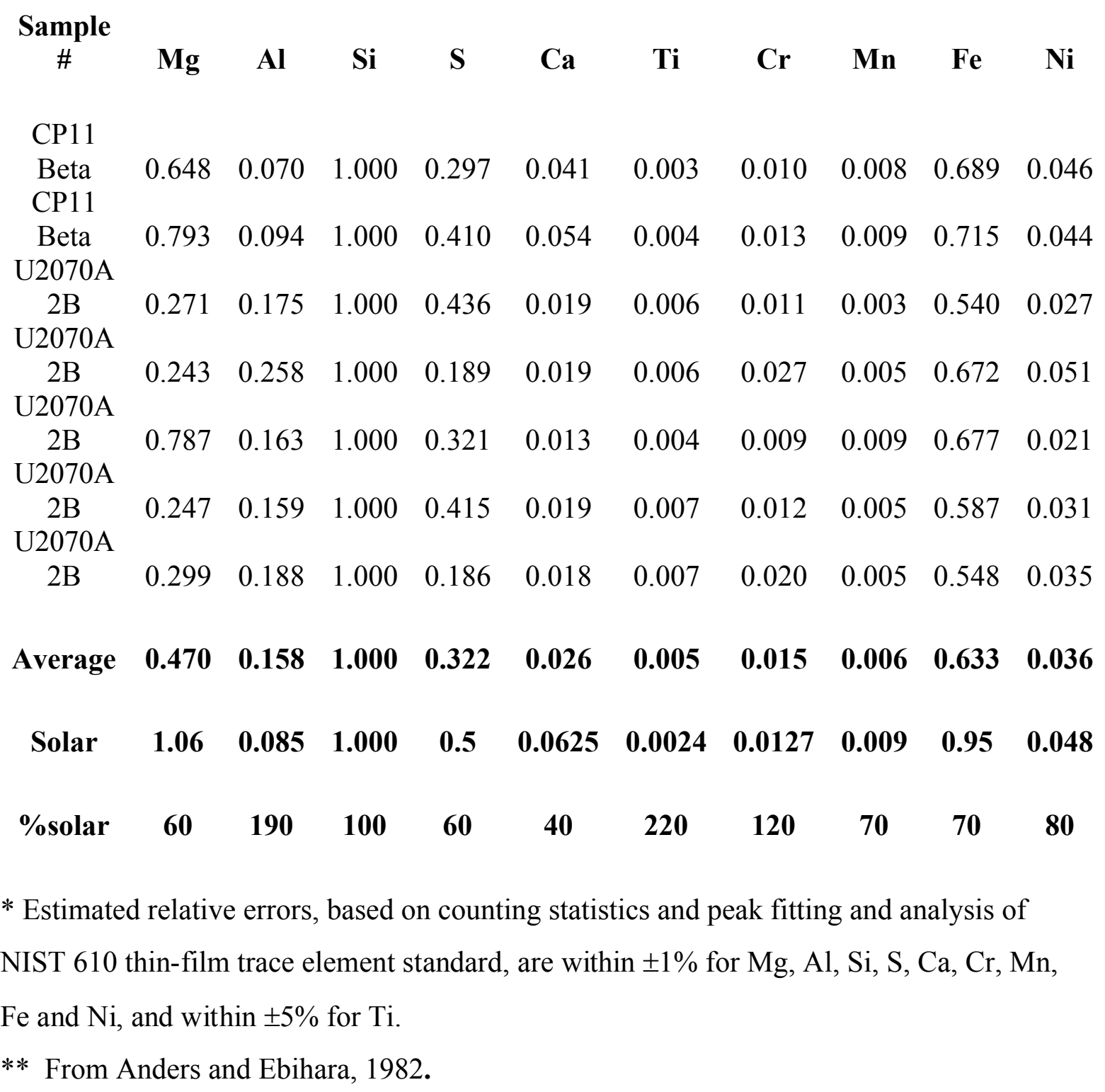



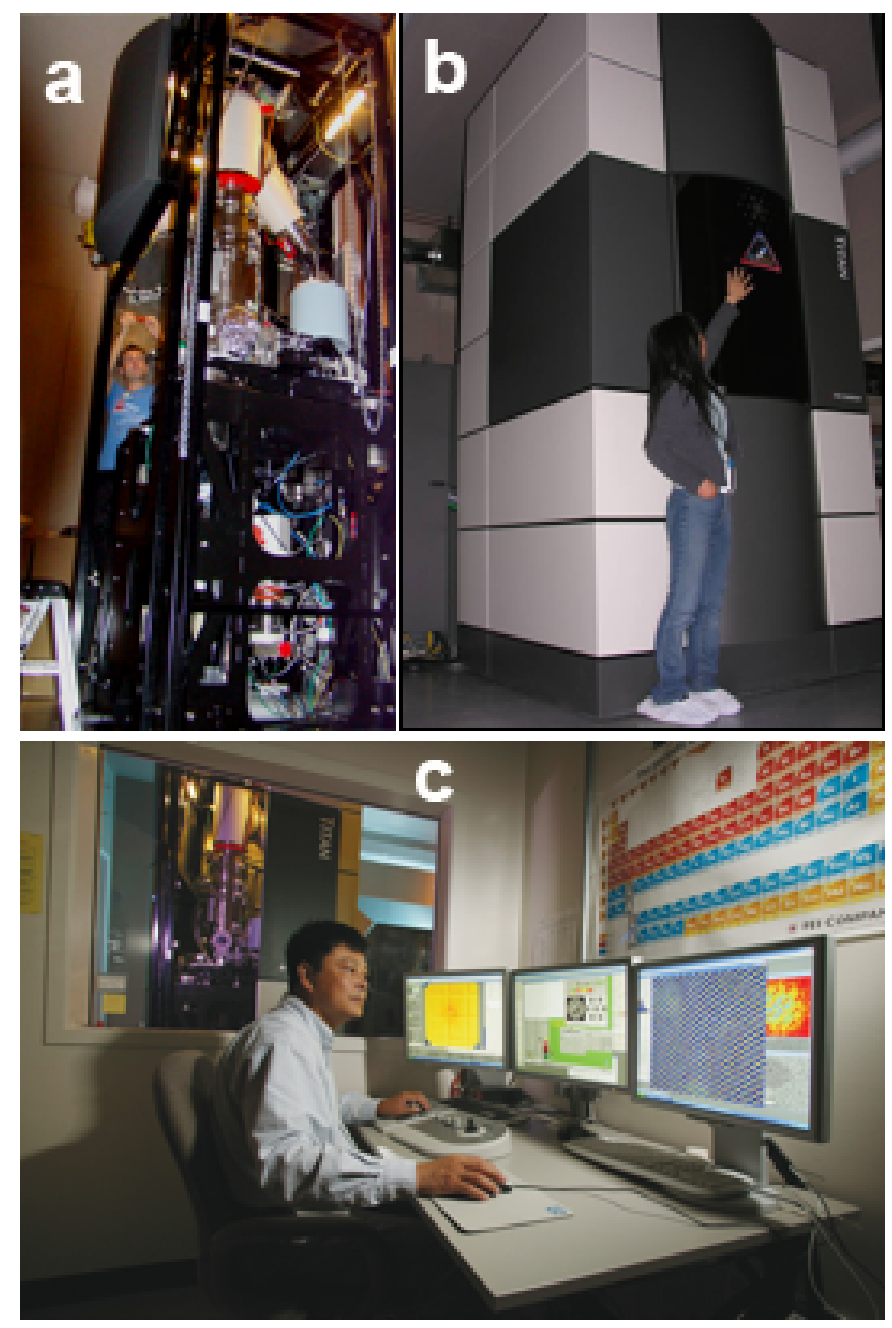

Figure 1. 

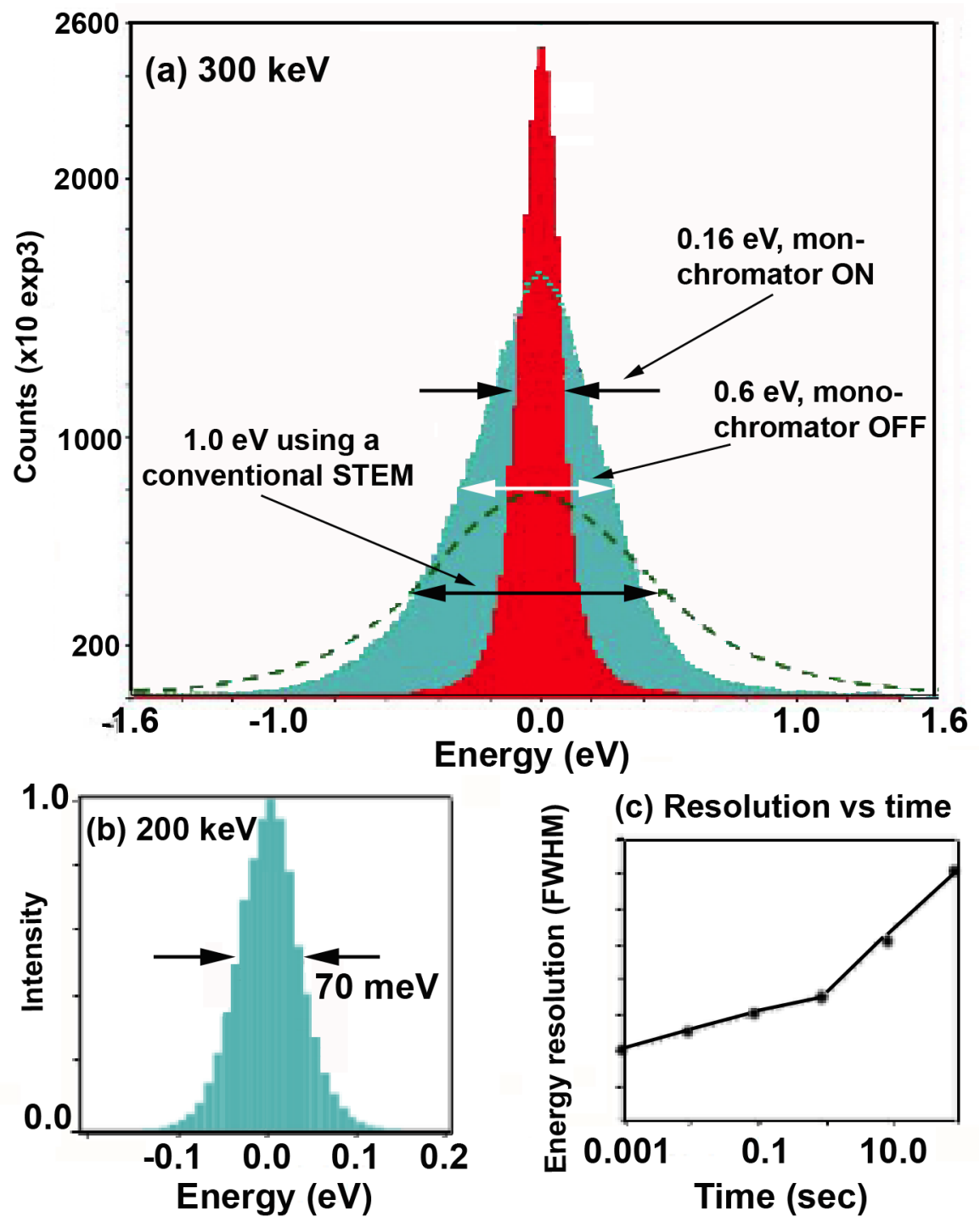

Figure 2. 


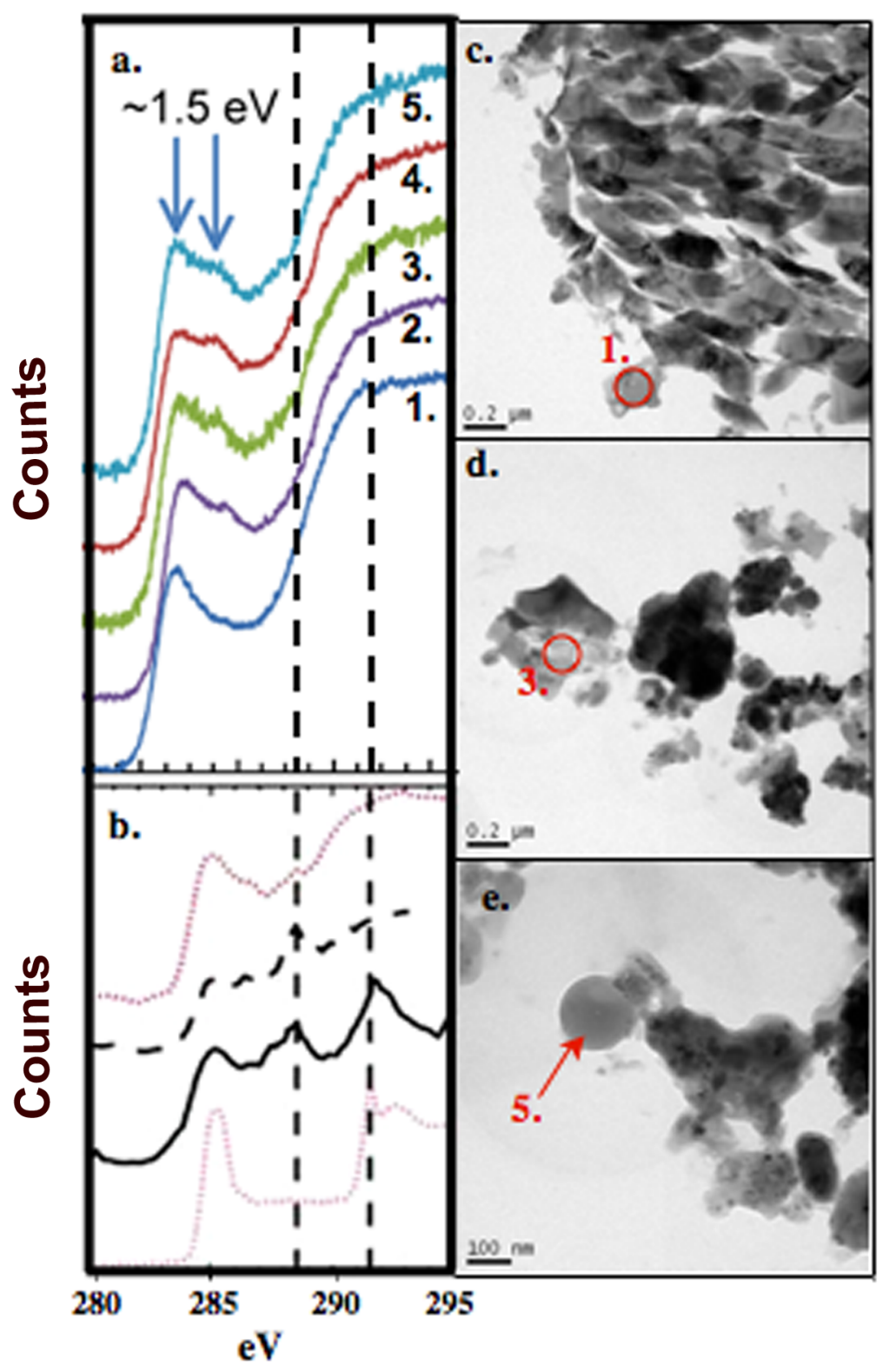

Figure 3. 


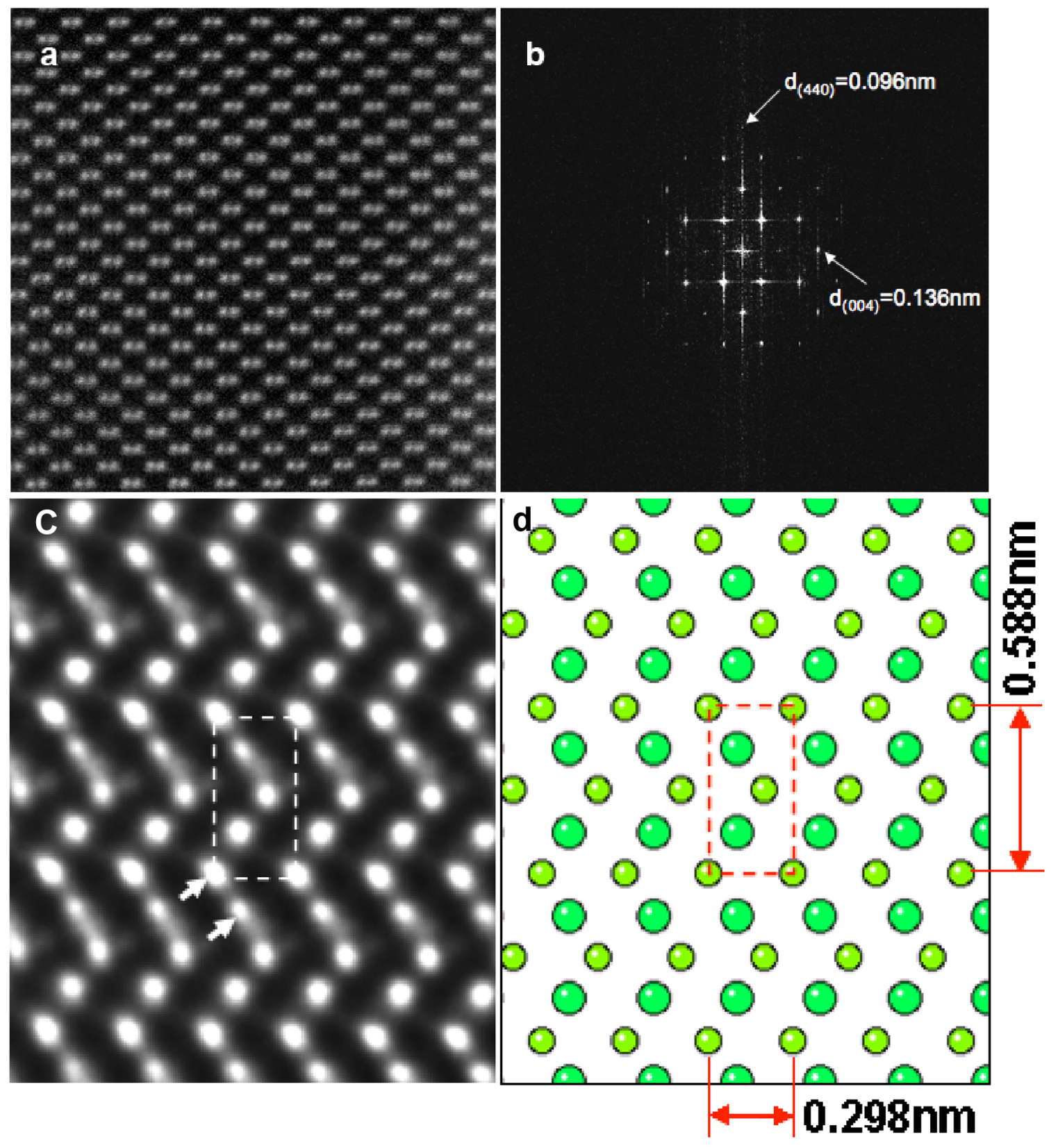

Figure 4. 

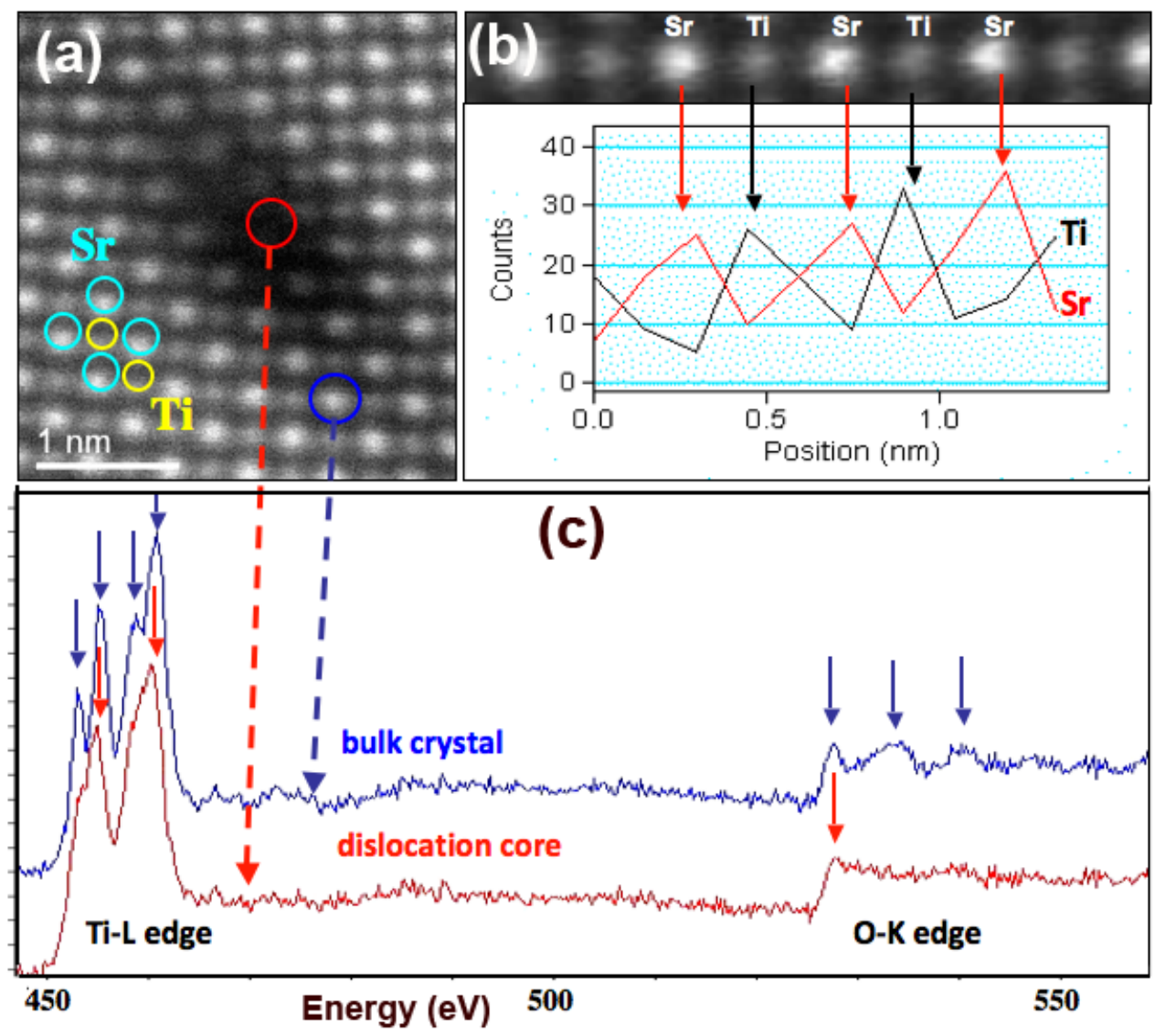

Figure 5. 

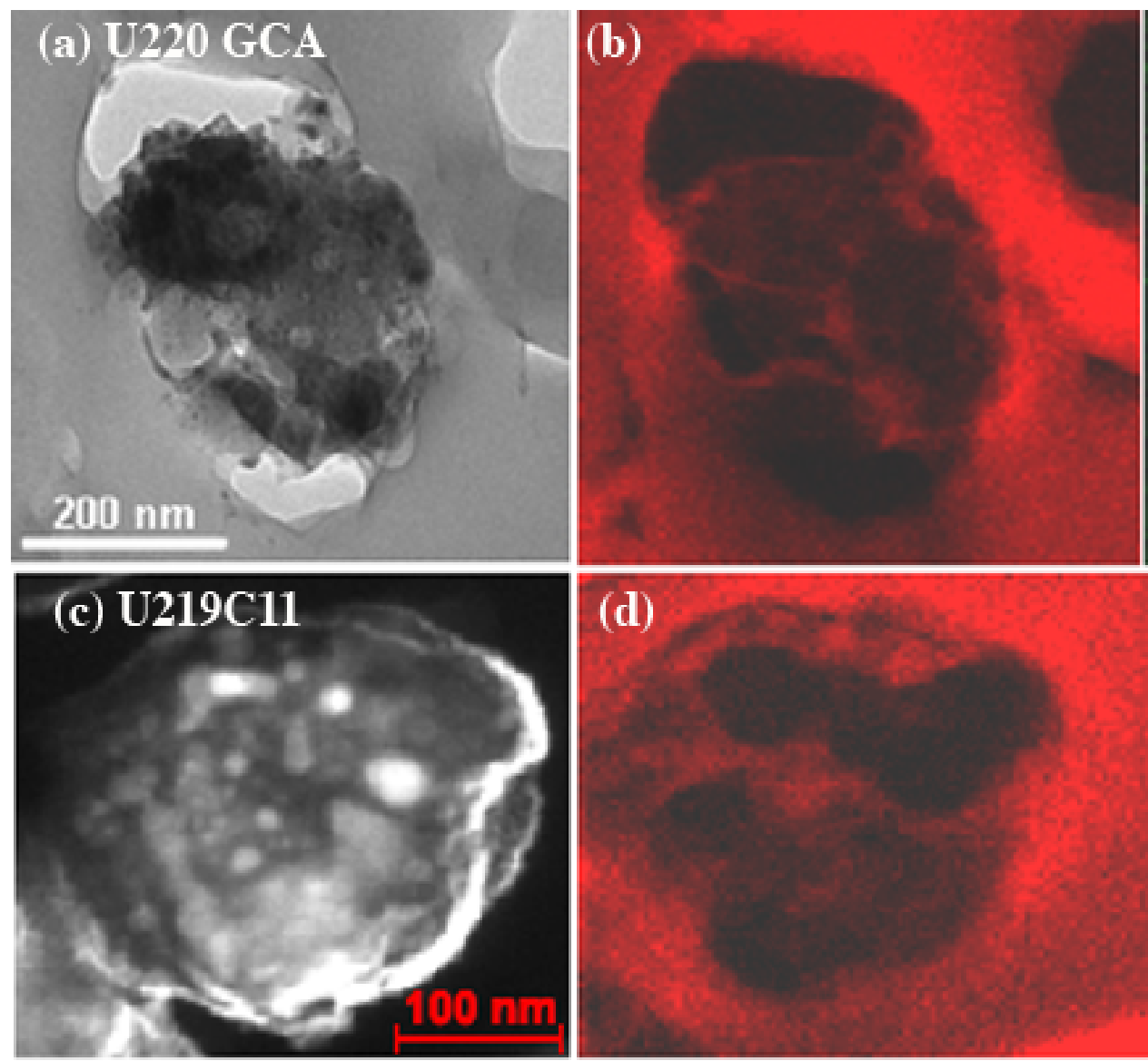

(d)

(e) $\mathrm{U} 219 \mathrm{C} 11$

(f)

$100 \mathrm{~nm}$

Figure 6 


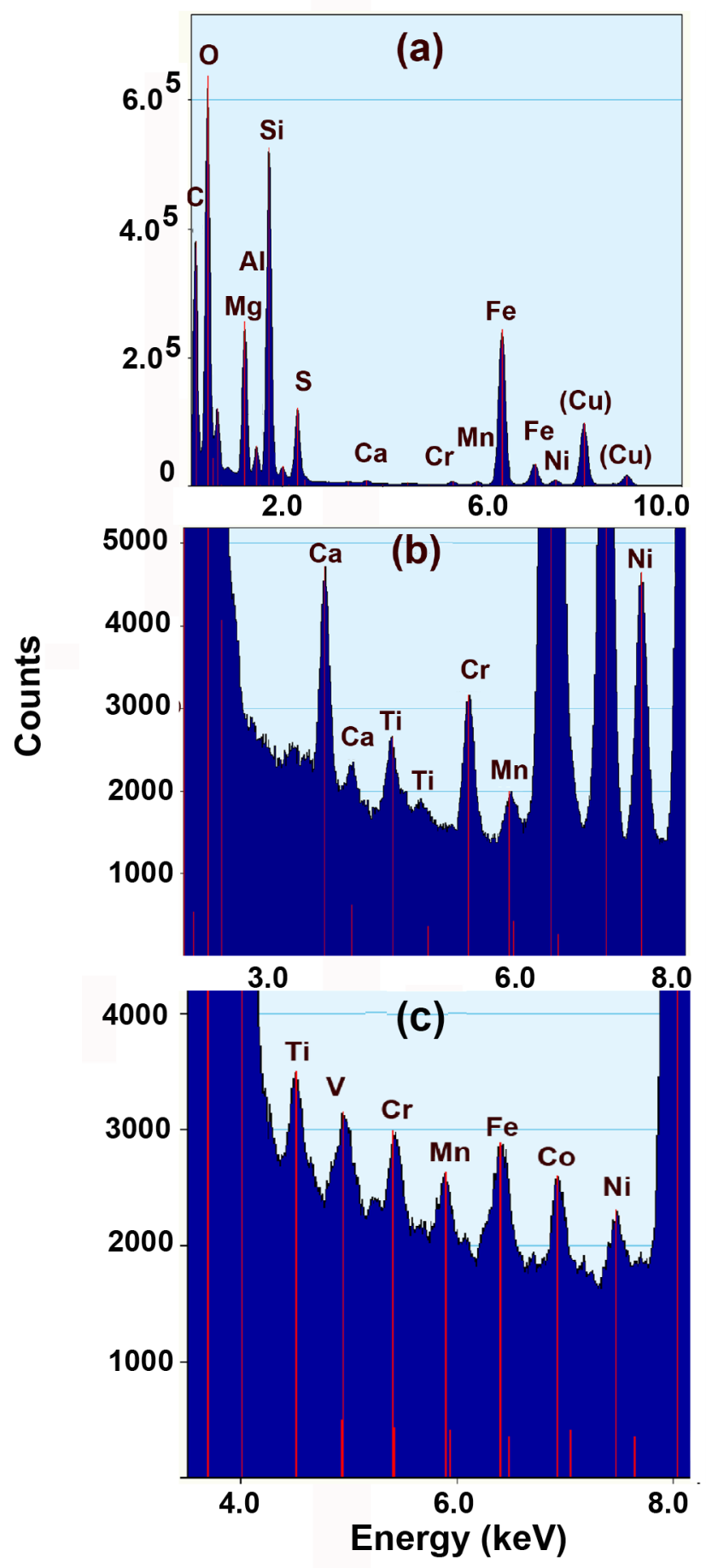

Figure 7. 

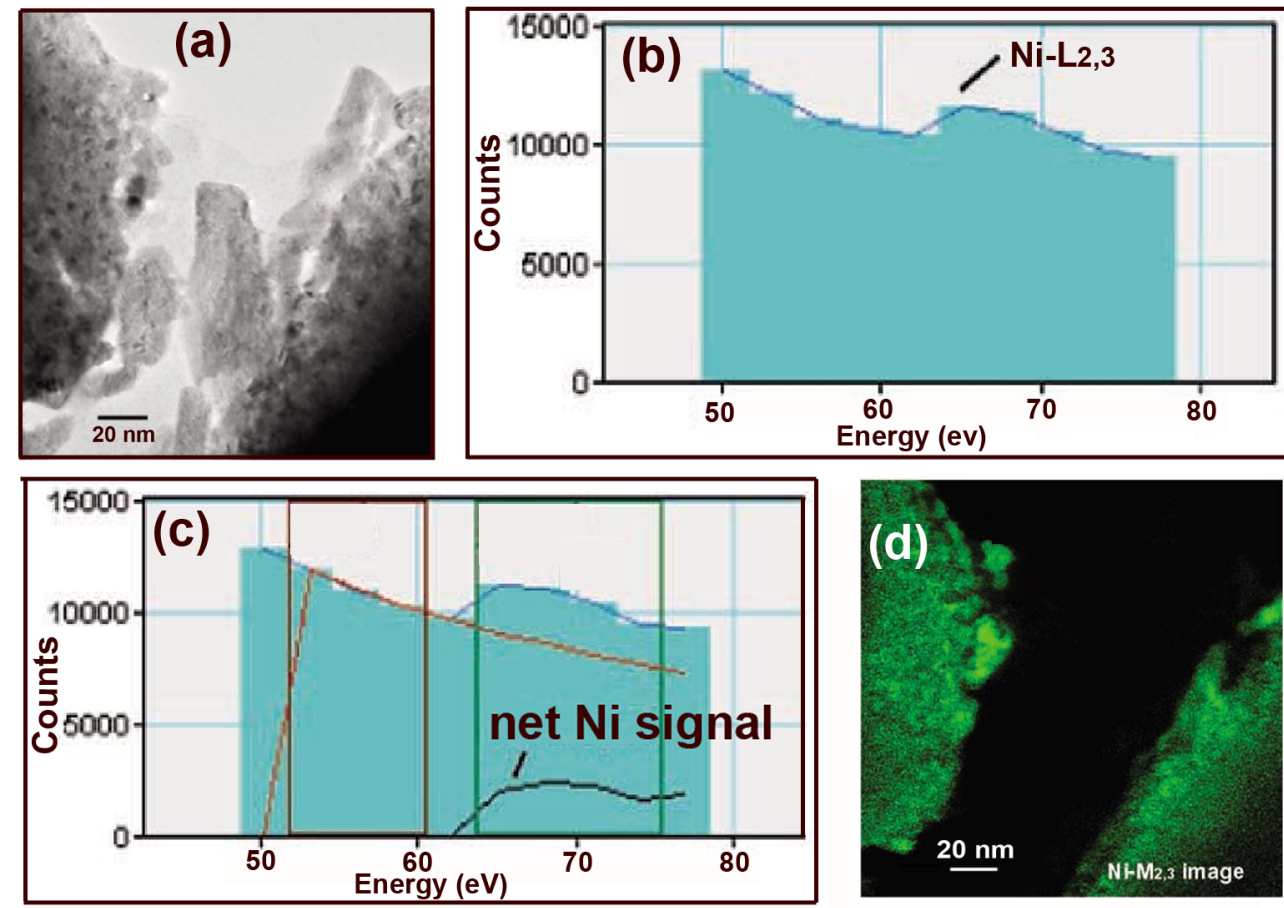

Figure 8. 


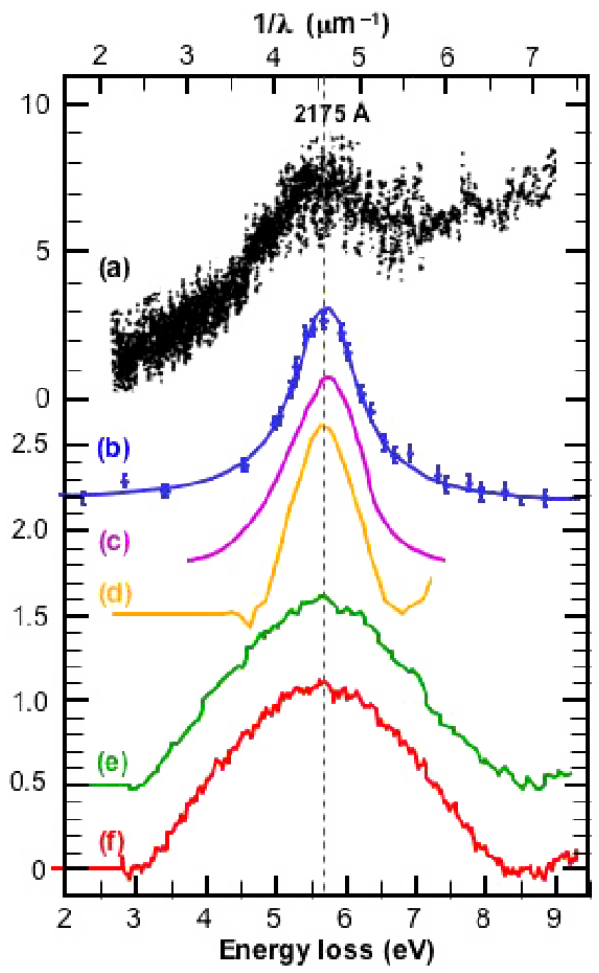

Figure 9. 

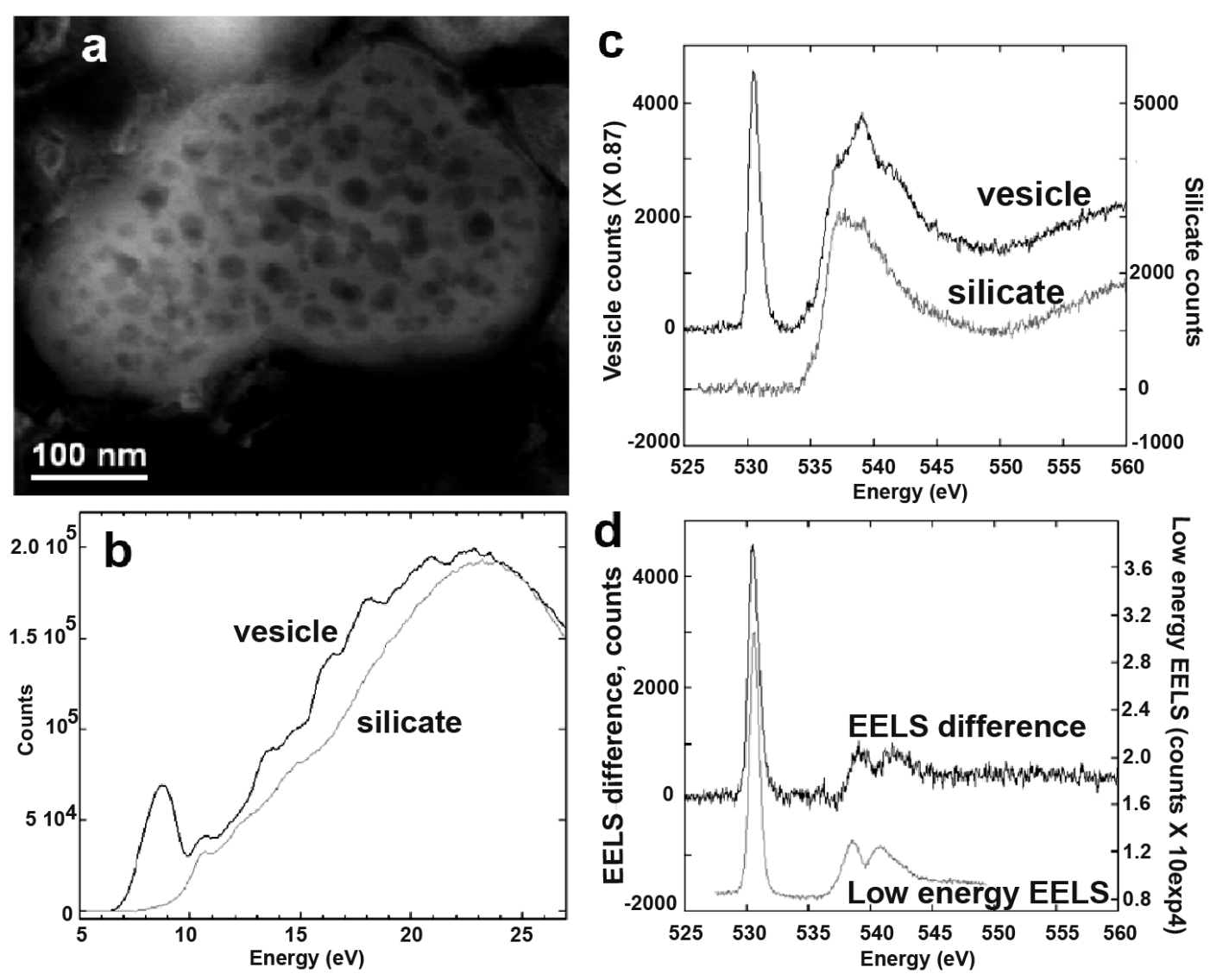

Figure 10. 


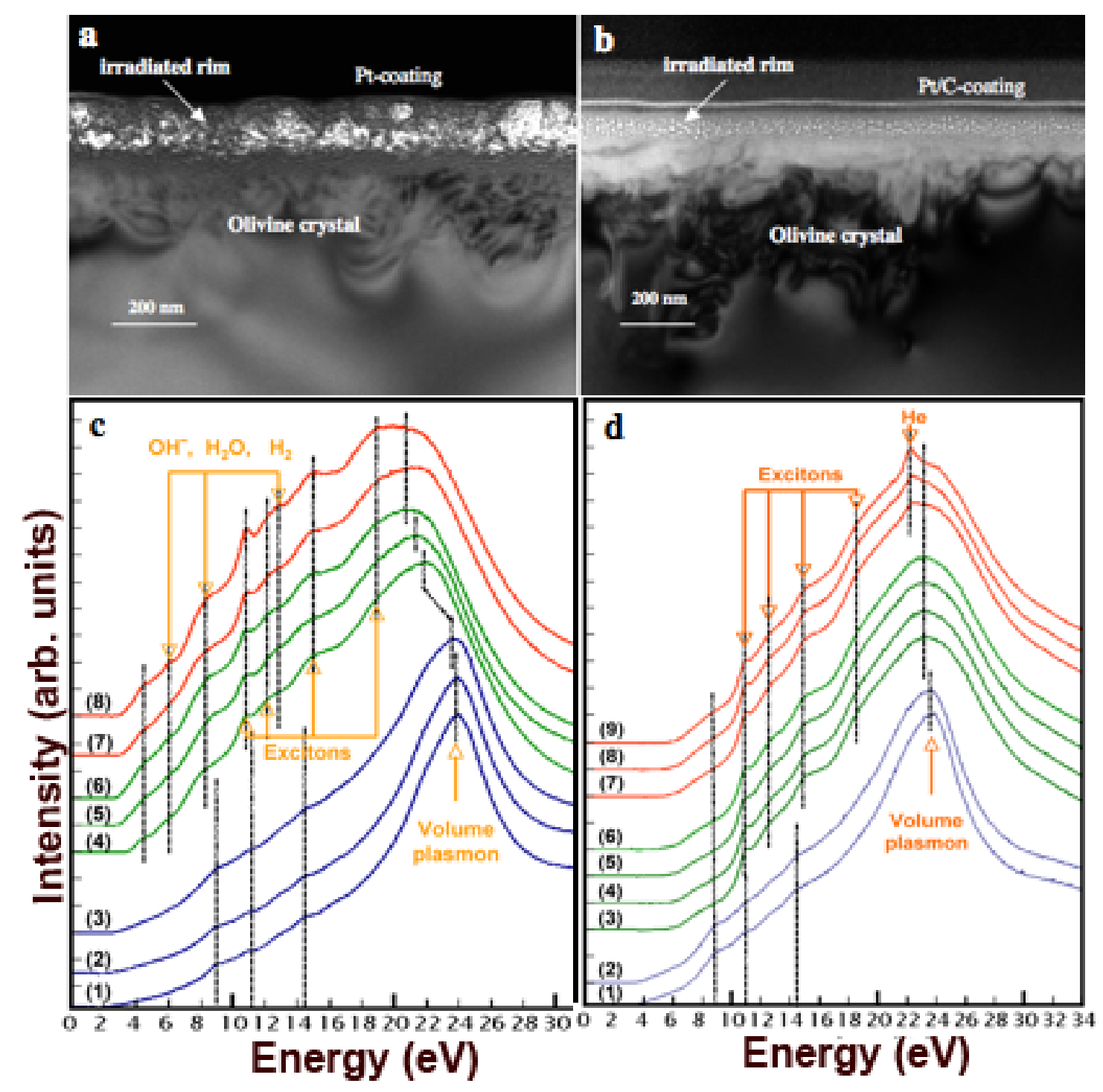

Figure 11. 